\title{
The geometry of solutions to a segregation problem for nondivergence systems
}

\author{
L. A. Caffarelli, A. L. Karakhanyan and Fang-Hua Lin
}

\begin{abstract}
Segregation systems and their singular perturbations arise in different areas: particle anihilation, population dynamics, material sciences. In this article we study the elliptic and parabolic limits of a nonvariational singularly perturbed problem. Existence and regularity properties of solutions and their limits are obtained.
\end{abstract}

Mathematics Subject Classification (2000). 35B25, 35B65, 35K55.

Keywords. Segregation of species, free boundary, nondivergence problem, singular point.

One of the simplest models for the segregation of species (or systems of particles that annihilate on contact) consists of setting a system of equations for the (vector) of nonnegative species densities $\vec{u}^{\varepsilon}=\left(u_{1}^{\varepsilon}, \ldots, u_{k}^{\varepsilon}\right)$, of the form

$$
L_{j}\left(u_{j}^{\varepsilon}\right)=\frac{1}{\varepsilon} F_{j}\left(\vec{u}^{\varepsilon}\right)
$$

where $L_{j}$ is a second order differential operator, $F_{j}$ vanishes if $u_{j}^{\varepsilon} u_{k}^{\varepsilon}=0$ for $k \neq j$ and it is strictly positive otherwise, forcing $u_{j}^{\varepsilon}$ to segregate $\left(u_{j}^{\varepsilon} u_{k}^{\varepsilon}\right.$ converge to zero) as $\varepsilon$ goes to zero.

In some applications, the system has a variational (or divergence) structure. For instance (see [CLLL], [CTV1])

$$
\Delta u_{j}^{\varepsilon}=\sum_{k \neq j} \frac{1}{\varepsilon} u_{j}^{\varepsilon}\left(u_{k}^{\varepsilon}\right)^{2}
$$

the Euler-Lagrange equations for vectors $\vec{u}$, stationary points of the functional

$$
\mathcal{E}(\vec{u})=\int\left(\sum_{j}\left(\nabla u_{j}\right)^{2}+\frac{1}{\varepsilon} \sum_{j, k}\left[u_{j}^{2} u_{k}^{2}\right]\right) .
$$

In others, e.g. in the case of this article (and of particle annihilation), the system is symmetric,

$$
\Delta u_{j}^{\varepsilon}=\sum_{k \neq j} \frac{1}{\varepsilon} u_{j}^{\varepsilon} u_{k}^{\varepsilon},
$$


and although it may appear to be a minimal change, its lack of variational structure imposes a different approach.

The final result is, though, very similar to those attained in [CL2], [CL3] for the variational case, mainly that the interface between any two components is smooth (the level set of a harmonic function), except in a "filament" (a set of Hausdorff dimension $n-2$ ), where three or more species may concur, mirroring the basic two-dimensional example given by

$$
w(x)=r^{3 / 2}\left|\cos \frac{3}{2} \theta\right|
$$

where each connected component of $\{w>0\}$ represents the support of a different species, and the three components concur at the origin.

This problem has received a considerable attention. See [CTV1]-[CTV4] for the discussion of the variational solutions and [CL2], [CL3] and [CLLL] for optimal partition problems. The system with a singular limit also appears in combustion theory related to flame propagation $[\mathrm{CR}],[\mathrm{BS}]$.

The parabolic version is not treated in the literature. In this paper we give a full description of the problem for the heat equation as a model case.

For the elliptic case we prove an improvement of the regularity result. We discuss the elliptic and parabolic versions separately. The paper is organized as follows: in the first section we show that the solutions $u^{\varepsilon}$ are uniformly Hölder continuous in $\varepsilon$, giving rise to a Hölder continuous vector $\vec{u}$ as a uniform limit as $\varepsilon$ goes to zero. Our approach works for more general classes of nonlinear uniformly elliptic and parabolic equations.

The vector $\vec{u}$ inherits several properties from $\vec{u}^{\varepsilon}$ that are the starting hypotheses of the regularity theory. In the next section we prove several properties of the limit function $\vec{u}=\lim _{\varepsilon \rightarrow 0} \vec{u}^{\varepsilon}$, such as harmonicity across the free boundary, regularity of $|\nabla u|^{2}$ across interfaces, and Lipschitz regularity. The latter is an application of a monotonicity formula introduced in $[\mathrm{ACF}]$.

The third section contains a geometric description of the free boundary and the proof of the clean-up lemma which states that a certain "flatness" implies regularity of the free boundary near a point where only two components concur.

Next we introduce Almgren's monotonicity formula [A] in order to find the structure of the free boundary near a singular point. The proof of Almgren's monotonicity formula for the heat equation is given in the Appendix.

\section{Uniform Hölder continuity for $\vec{u}^{\varepsilon}$}

We consider, in the ball $B_{1}$ of $\mathbb{R}^{n}$, a nonnegative solution, $u_{j}^{\varepsilon} \geq 0$, of the system

$$
\Delta u_{i}^{\varepsilon}=\frac{u_{i}^{\varepsilon}}{\varepsilon} \sum_{k \neq i} u_{k}^{\varepsilon} .
$$

For this section we may replace $\Delta u$ by a uniformly elliptic operator $L u=$ $D_{i}\left(a_{i j} D_{j} u\right)$ with bounded measurable coefficients $a_{i j}$. 
We will assume that the $u_{i}^{\varepsilon}$ are bounded $\left(0 \leq u_{i}^{\varepsilon} \leq M\right)$, and that $\vec{u}^{\varepsilon}=$ $\left(u_{1}^{\varepsilon}, \ldots, u_{m}^{\varepsilon}\right)$ is in $L^{1}$ or $L^{2}$, since being subharmonic (or $a$-subharmonic, i.e. $\left.L u_{i}^{\varepsilon} \geq 0\right)$ the $u_{i}^{\varepsilon}$ are bounded in $B_{1-h}$ for any sufficiently small $h>0$ by the mean value theorem.

Of course the $u_{i}^{\varepsilon}$ are smooth, with bounds depending on $\varepsilon$. Our first theorem is

Theorem 1. In $B_{1 / 2}$, for any $\varepsilon, \vec{u}^{\varepsilon}$ is $C^{\alpha}$ for some $\alpha>0$ independent of $\varepsilon$, and

$$
\left\|\vec{u}^{\varepsilon}\right\|_{C^{\alpha}\left(B_{1 / 2}\right)} \leq C(M),
$$

with $C(M)$ also independent of $\varepsilon$.

Remark. For this first theorem we may replace the Laplacian by any other operator $L u$, linear elliptic or parabolic with the following three properties:

Let $w$ satisfy $L w=f \geq 0$ and

$$
\underset{B_{2}}{\operatorname{osc}} w=\sup _{B_{2}} w-\inf _{B_{2}} w=1 .
$$

Then for a positive constant $\mu\left(\gamma_{0}\right)$ depending on $\gamma_{0}$ one has:

(a) If $\left|\left\{f \geq \gamma_{0}>0\right\}\right| \geq \gamma_{0}>0$ then

$$
\sup _{B_{1}} w \leq \sup _{B_{2}} w-\mu\left(\gamma_{0}\right) .
$$

(b) If $\left|\left\{w \leq \sup w-\gamma_{0}\right\}\right| \geq \gamma_{0}$ then

$$
\sup _{B_{1}} w \leq \sup _{B_{2}} w-\mu\left(\gamma_{0}\right) .
$$

(c) If $|f| \ll \operatorname{osc}_{B_{2}} w=1$, then

$$
\underset{B_{1}}{\operatorname{Osc}} w \leq \underset{B_{2}}{\operatorname{Osc}} w-\gamma_{0}
$$

This is true for uniformly elliptic or parabolic equations with bounded measurable coefficients from De Giorgi-Nash-Moser (and the Littman-StampacchiaWeinberger estimate [LSW]) for divergence equations, and the Aleksandrov-Bakelman-Pucci and Krylov-Safonov theory for nondivergence equations. In the parabolic case we must take consecutive parabolic cylinders.

In order to prove Theorem 1 we first state the following

Lemma 2. Let $m_{i}(R)=\min _{B_{R}} u_{i}^{\varepsilon}, M_{i}(R)=\max _{B_{R}} u_{i}^{\varepsilon}$, and $\mathcal{O}_{i}(R)=\operatorname{osc}_{B_{R}} u_{i}^{\varepsilon}$. Suppose that either of the following is satisfied for some positive constant $\gamma_{0}$ :

(a) $\left|\left\{x \in B_{1 / 4}: u_{i}^{\varepsilon}(x) \leq M_{i}-\gamma_{0} \mathcal{O}_{i}\right\}\right| \geq \gamma_{0}$ where $m_{i}=m_{i}(1), M_{i}=M_{i}(1)$, $\mathcal{O}_{i}=\mathcal{O}_{i}(1)$,

(b) $\left|\left\{x \in B_{1 / 4}: \Delta u_{i}^{\varepsilon}(x) \geq \gamma_{0} \mathcal{O}_{i}\right\}\right| \geq \gamma_{0}$,

(c) $\left|\left\{x \in B_{1 / 4}: \Delta u_{i}^{\varepsilon} \geq \gamma_{0} u_{i}^{\varepsilon}\right\}\right| \geq \gamma_{0}$.

Then there exists a small positive constant $c_{0}=c_{0}\left(\gamma_{0}\right)$ such that the following decay estimate is valid:

$$
M_{i}(1 / 4) \leq M_{i}-c_{0} \mathcal{O}_{i}
$$


Proof. It is well-known that if $L u \geq 0$ in $D \subset B_{R}$ where $u \geq K$ in $D$ and $u=K$ on $\partial D \cap B_{R}$ then

$$
\sup _{D \cap B_{R / 4}} u \leq \frac{1}{1+C \frac{\left|B_{R} \cap D^{c}\right|}{R^{n}}} \cdot \sup _{D} u .
$$

This estimate is classical (see [La]) and as an application to our problem

$$
\Delta u_{i}^{\varepsilon}=\frac{u_{i}^{\varepsilon} \sum_{j \neq i} u_{j}^{\varepsilon}}{\varepsilon} \quad \text { in } B_{1}
$$

with $u=u_{i}^{\varepsilon}$ we have

$$
M_{i}(1 / 4)=\sup _{B_{1 / 4}} u_{i}^{\varepsilon} \leq \max \left(\frac{M_{i}}{1+C \gamma_{0}}, M_{i}-\gamma_{0}\left(M_{i}-m_{i}\right)\right)=c_{0} M_{i}, \quad c_{0}<1 .
$$

In particular, $\operatorname{osc}_{B_{R}} u_{i}^{\varepsilon}=\mathcal{O}_{i}(R)$ decays and

$$
\mathcal{O}_{i}(1 / 4)=M_{i}(1 / 4)-m_{i}(1 / 4) \leq c_{0} M_{i}-m_{i}(1 / 4) \leq c_{0}\left(M_{i}-m_{i}\right)=c_{0} \mathcal{O}_{i}(1)
$$

since $m_{i}(1 / 4) \geq m_{i}(1) \geq c_{0} m_{i}, c_{0}<1$ so in case (a) the conclusion follows.

To prove case (b) we use Green's representation formula

$$
u_{i}^{\varepsilon}(x)=v_{i}^{\varepsilon}(x)-\int_{B_{1}} G(x, y) \Delta u_{i}^{\varepsilon}(y) d y, \quad G(x, y) \geq 0,
$$

where $v_{i}^{\varepsilon}$ is the harmonic replacement of $u_{i}^{\varepsilon}$ in $B_{1}$, and $G(x, y)$ is the Green's function of $B_{1}$. Then we have

$$
\begin{aligned}
0 \leq v_{i}^{\varepsilon}(x)-u_{i}^{\varepsilon}(x) & =\int_{B_{1}} G(x, y) \Delta u_{i}^{\varepsilon}(y) d y \geq \int_{B_{1 / 4} \cap\left\{\Delta u_{i}^{\varepsilon} \geq \gamma_{0} \mathcal{O}_{i}\right\}} G(x, y) \Delta u_{i}^{\varepsilon}(y) d y \\
& \geq \gamma_{0} \mathcal{O}_{i} \int_{B_{1 / 4} \cap\left\{\Delta u_{i}^{\varepsilon} \geq \gamma_{0} \mathcal{O}_{i}\right\}} G(x, y) d y \geq C \gamma_{0}^{2} \mathcal{O}_{i},
\end{aligned}
$$

that is, $u_{i}^{\varepsilon}(x) \leq v_{i}^{\varepsilon}(x)-C \gamma_{0}^{2} \mathcal{O}_{i}(1)$ and $M_{i}(1 / 4) \leq c_{0} M_{i}$ for $c_{0}<1$.

To handle (c), consider $A_{i}=\left\{x \in B_{1 / 4}: \Delta u_{i}^{\varepsilon} \geq \gamma_{0} u_{i}^{\varepsilon}\right\}$ and $H_{i}=\left\{x \in A_{i}\right.$ : $\left.u_{i}^{\varepsilon}(x)<M_{i} / 2\right\}$. First let us assume that

$$
\left|A_{i} \backslash H_{i}\right| \geq \frac{1}{2}\left|A_{i}\right| .
$$

Then

Therefore

$$
\left\{x \in A_{i}: u_{i}^{\varepsilon}(x) \geq M_{i} / 2\right\} \subset\left\{\Delta u_{i}^{\varepsilon} \geq \gamma_{0} M_{i} / 2\right\} .
$$

$$
\left|\left\{\Delta u_{i}^{\varepsilon} \geq \gamma_{0} M_{i} / 2\right\}\right| \geq\left|\left\{x \in A_{i}: u_{i}^{\varepsilon}(x) \geq M_{i} / 2\right\}\right|=\left|A_{i} \backslash H_{i}\right| \geq \frac{1}{2}\left|A_{i}\right| \geq \frac{1}{2} \gamma_{0}
$$

by (1.1). So part (b) applies and we have

$$
M_{i}(1 / 4) \leq c_{0} M_{i}(1), \quad c_{0}<1 .
$$

Now assume that

$$
\left|A_{i} \backslash H_{i}\right| \leq \frac{1}{2}\left|A_{i}\right|
$$


Then

$$
\left|H_{i}\right|=\left|A_{i} \backslash\left(A_{i} \backslash H\right)\right|=\left|A_{i}\right|-\left|A_{i} \backslash H_{i}\right| \geq \frac{1}{2}\left|A_{i}\right| \geq \frac{1}{2} \gamma_{0}
$$

by (1.2). This implies $\left|\left\{x \in B_{1 / 4}: u_{i}^{\varepsilon}(x) \geq M_{i} / 2\right\}\right| \geq\left|H_{i}\right| \geq \gamma_{0} / 2$, and from (a) the result follows.

We now return to the proof of the Hölder regularity. To simplify the notations we shall denote $\vec{u}^{\varepsilon}$ by $u$.

Proof. The proof is inductive, based on reducing the oscillation of the vector $u$ in consecutive balls $B_{\lambda^{k}}$ by a fixed constant $\mu<1$, for some (fixed) $\lambda<1$. Since we can always renormalize the system to the unit ball by

$$
u^{*}(x)=\frac{1}{M} u\left(\lambda^{k} x\right)
$$

with $M=\sup _{j, x} u_{j}$ where $x \in B_{\lambda^{k}}$ into the same system (with a different $\varepsilon$ ), it is enough to show that the largest of the individual oscillations decays from $B_{1}$ to $B_{\lambda}$, for a system $u$, with $\max _{j, x} u_{j}(x)=1$ on $B_{1}$. Let $\mathcal{O}_{j}=\operatorname{osc}_{B_{1}} u_{j}$ and without loss of generality assume that $1 \geq \mathcal{O}_{1} \geq \cdots \geq \mathcal{O}_{k}$.

We start with several simple cases in which the oscillation of a given component decreases by a fixed proportion (see Lemma 2):

(a) If $m_{i} \leq u_{i} \leq M_{i}$ and $\left|\left\{u_{i} \leq M_{i}-\gamma_{0}\left(M_{i}-m_{i}\right)\right\}\right| \geq \gamma_{0}$ then in $B_{1 / 2}, M_{i}$ decays to $M_{i}-\mu\left(\gamma_{0}\right)\left(M_{i}-m_{i}\right)$ and $\mathcal{O}_{i}$ decays to $\left[1-\mu\left(\gamma_{0}\right)\right] \mathcal{O}_{i}$.

(b) If $\left|\left\{L u_{i} \geq \gamma_{0}\left(M_{i}-m_{i}\right)\right\}\right| \geq \gamma_{0}>0$, then again $M_{i}$ and $\mathcal{O}_{i}$ decay by amount proportional to $\mathcal{O}_{i}$

(c) If $|A|=\left|\left\{L u_{i} \geq \gamma_{0} u_{i}\right\}\right| \geq \gamma_{0}$, then either $u_{i} \geq M_{i} / 2$ half of the time in $A$ and $M_{i}$ decays from (b), or $u_{i} \leq M_{i} / 2$ and $M_{i}$ decays from (a) (in both cases the amounts of decays in $M_{i}$ are proportional to $\mathcal{O}_{i}$ ).

(d) If $\sum_{j>1} \mathcal{O}_{j} \leq \delta \mathcal{O}_{1}, \delta>0$, then we let $w$ be the solution of $L w=0,\left.w\right|_{\partial B_{1}}$
$=u_{1}$.

Since $u_{1}+\sum_{j>1}\left(M_{j}-u_{j}\right)$ is a supersolution, we have

$$
u_{1} \leq w \leq u_{1}+\sum_{j>1}\left(M_{j}-u_{j}\right) \leq u_{1}+\delta \mathcal{O}_{1} .
$$

But

$$
\underset{B_{1 / 2}}{\operatorname{Osc}} w \leq(1-\mu) \underset{B_{1}}{\operatorname{osc}} w \leq \mu_{1} \mathcal{O}_{1}
$$

hence

$$
\underset{B_{1 / 2}}{\operatorname{OSc}} u_{1} \leq((1-\mu)+\delta) \mathcal{O}_{1} \leq \mathcal{O}_{1}
$$

and osc $u_{1}$ decreases proportionally to $\mathcal{O}_{1}$.

Therefore, to establish our basic iterative decay estimate for oscillations, it is sufficient to prove that either

$(\alpha)$ among those $\mathcal{O}_{j}$ 's with $\mathcal{O}_{j} \geq \delta \mathcal{O}_{1}$ there is at least one that decays by a factor whenever the sizes of balls shrink by a half; or

$(\beta)$ all $\mathcal{O}_{j}$ 's with possible exception of $\mathcal{O}_{1}$ decay by a factor. 
Indeed, applying $(\alpha)$ or $(\beta)$ a finite number of times we will force all $\mathcal{O}_{j}$ bigger than $\delta \mathcal{O}_{1}$ to decrease.

Case 1: We first discuss the case $\varepsilon>1$, i.e., for $\theta=1 / \varepsilon$ :

$$
\left\{\begin{array}{l}
0 \leq u_{j} \leq 1, \\
\Delta u_{i}=\theta u_{i} \sum_{j \neq i} u_{j} \text { with } \theta<1, \\
\max u_{j_{0}}=1 \text { for some } j_{0} .
\end{array}\right.
$$

Consider two subcases: (i) $\mathcal{O}_{1} \sim 1$, i.e., $\mathcal{O}_{1} \geq \delta_{0}$ and (ii) $\mathcal{O}_{1} \ll 1$.

We first consider (i). If $\operatorname{osc}_{B_{1 / 2}} u_{1}=\mathcal{O}_{1}$ has not decreased, so that $\mathcal{O}_{1} \geq$ $\left(1-\gamma_{0}\right) \mathcal{O}_{1}$, then

$$
\bar{m}_{1}=\min _{B_{1 / 2}} u_{1} \leq M_{1}-\mathcal{O}_{1} \leq M_{1}-\left(1-\gamma_{0}\right) \mathcal{O}_{1} .
$$

Note that there is a point $x_{0}$ in $B_{1 / 2}$ such that $u_{1}\left(x_{0}\right)=\bar{m}_{1}$.

Since the right hand side of the equation is $<1, u_{1}(x) \leq \bar{m}_{1}+\frac{1}{2} \delta_{0}$ in a neighborhood of size $\delta_{0}$, i.e., for $x$ in $B_{\delta_{0}}\left(x_{0}\right)$ one has

$$
u_{1}(x) \leq M_{1}-\left(1-\gamma_{0}\right) \mathcal{O}_{1}+\frac{1}{2} \delta_{0} \leq M_{1}\left(1-\delta_{0}\right)
$$

From observation $(\mathrm{a}), \mathcal{O}_{i}(1 / 2)$ has decreased proportionally to $\mathcal{O}_{i}$. We thus obtain a contradiction.

Now consider (ii) $\mathcal{O}_{1} \ll 1$. Again we divide it into two subcases. Assume first that all $\mathcal{O}_{j} \leq \frac{1}{2} M_{j}$ (i.e., $\frac{1}{2} M_{j} \leq u_{j} \leq M_{j}$ for all $j$ ). Then the right hand side of the $j$-th equation is $\sim \theta M_{j}$ for $j \neq j_{0}\left(u_{j_{0}} \sim 1\right)$.

If $\theta M_{j} \geq \gamma_{0} \mathcal{O}_{j}$ for some $\gamma_{0}$ small, we have decay from observation (b).

If $\theta M_{j} \leq \gamma_{0} \mathcal{O}_{j}$, we have decay from regularity (the right hand side is much smaller than the oscillation).

On the other hand, if for some $j\left(j \neq j_{0}\right), \mathcal{O}_{j} \geq \frac{1}{2} M_{j}$, then

$$
L u_{j} \sim \theta u_{j} \leq 2 \theta \mathcal{O}_{j}
$$
$\ll \mathcal{O}_{j}$.

If $\theta \ll 1$ we have decay of $\mathcal{O}_{j}$ from regularity since the right hand side is

If $\theta \sim 1$, we have it from observation (c).

Case 2: We now go to the case $\varepsilon<1$. We consider two subcases: $M_{1} \geq \varepsilon$ and $M_{1}<\varepsilon$.

Suppose $M_{1} \geq \varepsilon$. If $\mathcal{O}_{1}$ does not decay, we must have (from Lemma $2(\mathrm{a})$ )

$$
\left|\left\{u_{1} \geq \varepsilon / 2\right\}\right| \geq 1 / 2 \text {. }
$$

Then, for $i \neq 1$,

$$
\left|\left\{L u_{i} \geq u_{i} / 2\right\}\right| \geq 1 / 2 .
$$

Thus all $M_{i}$ decay for $i \neq 1$. 
Now suppose $M_{1}$ and hence all $\mathcal{O}_{j}$ are smaller than $\varepsilon$ (since $\left.\mathcal{O}_{j} \leq \mathcal{O}_{1} \leq M_{1}\right)$. Since $M_{j_{0}}=1$, we have $u_{j_{0}} \geq 1-\varepsilon$, so

$$
L u_{1} \geq \frac{1}{\varepsilon} u_{1},
$$

and (b) applies.

The proof is complete.

Corollary 3. Given a family of solutions $(\vec{u})^{\varepsilon_{k}}$, with $\varepsilon_{k}$ going to zero, there is a subsequence that converges uniformly to a $C^{\alpha}$ function $\vec{u}$.

\section{General properties of the limit $u$}

We now restrict ourselves to the Laplace operator.

Lemma 4. Let $\vec{u}(x)=\left(u_{1}(x), \ldots, u_{m}(x)\right)$ be the limit function from Corollary 3 . Then:

(i) $\Delta u_{i}$ is a positive measure and

$$
\Delta u_{i} \leq \sum_{j \neq i} \Delta u_{j}
$$

(ii) $\Delta u_{i}=0$ whenever $u_{i}>0$

Proof. (i) follows from the fact that all $u_{i}^{\varepsilon}$ are subharmonic and for each $\varepsilon$, and a nonnegative function $\varphi \in C_{0}^{\infty}(\Omega)$,

$$
\int(\Delta \varphi) u_{i}^{\varepsilon}=\int \varphi \Delta u_{i}^{\varepsilon} \leq \int \varphi\left(\sum_{j \neq i} \Delta u_{j}^{\varepsilon}\right)=\int(\Delta \varphi) \sum_{j \neq i} u_{j}^{\varepsilon} .
$$

To prove (ii) we will use the formula

$$
f_{\partial B_{r}(x)}\left[u(y)-u\left(x_{0}\right)\right]=\int_{0}^{r}\left(\int_{B_{\rho}} \Delta u\right) \frac{d \rho}{\rho^{n-1}} \geq r^{2} f_{B_{r}} \Delta u .
$$

By Hölder continuity, if $u_{1}^{\varepsilon}\left(x_{0}\right)=\alpha_{0}>0$, then $\left|u_{1}^{\varepsilon}(y)-u_{1}^{\varepsilon}\left(x_{0}\right)\right| \leq \alpha_{0} / 2$ in a neighborhood $B_{h}\left(x_{0}\right)$. Then

$$
f_{B_{h}} u_{1}^{\varepsilon}\left(\frac{1}{\varepsilon} \sum_{j \neq 1} u_{j}^{\varepsilon}\right)=f_{B_{h}} \Delta u_{1}^{\varepsilon} \leq \frac{\alpha_{0}}{2 h^{2}}
$$

for $\varepsilon$ small, from the uniform convergence. Since $u_{1}^{\varepsilon} \geq \alpha_{0} / 2$ in $B_{h}$, we get

$$
f_{B_{h}} \frac{1}{\varepsilon}\left(\sum_{j \neq 1} u_{j}^{\varepsilon}\right) \leq \frac{1}{h^{2}} .
$$

As $\varepsilon \rightarrow 0, \sum_{j \neq 1} u_{j}^{\varepsilon}$ goes to zero.

Corollary 5. The Hölder continuous functions $u_{j}$ have disjoint supports and are harmonic when positive. 
In order to show the linear decay of $u_{i}$ away from the boundary of its support, we recall the monotonicity formula introduced in $[\mathrm{ACF}]$ (see [CSa] for details).

Corollary 6. Let $v_{1}$ and $v_{2}$ be defined as

$$
v_{1}=\sum_{j=1}^{j_{0}} u_{j}, \quad v_{2}=\sum_{j=j_{0}+1}^{k} u_{j},
$$

and let $x_{0}$ be a point on the boundary of $\operatorname{supp} u_{j_{0}}$. Then $J(R)=J_{R}\left(v_{1}, v_{2}\right)=$ $D\left(v_{1}, R\right) D\left(v_{2}, R\right) \nearrow$ as $R \nearrow$, where $D(v, R)$ denotes the Dirichlet average

$$
D(v, R)=\frac{1}{R^{2}} \int_{B_{R}\left(x_{0}\right)} \frac{(\nabla v)^{2}}{\left|x-x_{0}\right|^{n-2}} d x .
$$

Furthermore,

$$
\left(\frac{1}{R} f_{\partial B_{R}} v_{i}\right)^{2} \leq C D\left(v_{i}, R\right) \leq \frac{C}{R^{2}} f_{B_{2 R} \backslash B_{R}} v_{i}^{2}, \quad i=1,2 .
$$

Proof. The second inequality in (2.1) follows from [CSa, (12.16)]. For the proof of the first inequality we refer to $[\mathrm{ACF}]$.

Lemma 7 (Linear decay of $u$ at the boundary of its support). Let $x_{0} \in B_{1 / 2} \cap$ $\partial \operatorname{supp}\left(u_{1}\right)$. Then

$$
\begin{aligned}
& \text { (a) } \frac{1}{R} f_{B_{R}\left(x_{0}\right)} u_{1} \leq C J_{R}\left(u_{1}, \sum_{j \neq 1} u_{j}\right) \leq C\|u\|_{L^{2}\left(B_{1 / 2}\right)} \text {, } \\
& \text { (b) } \sup _{B_{R}} u_{1} \leq C R .
\end{aligned}
$$

Proof. $u_{1}-\sum_{j \neq 1} u_{j}$ is superharmonic. Since $u_{j}\left(x_{0}\right)=0$ for all $j$,

$$
f_{\partial B_{R}} u_{1} \leq f_{\partial B_{R}} \sum_{j \neq 1} u_{j}
$$

Thus

and also

$$
\theta_{R}=\frac{1}{R} f_{\partial B_{R}} u_{1} \leq C\left[D\left(u_{1}, R\right)\right]^{1 / 2}
$$

$$
\theta_{R} \leq \frac{1}{R} f_{\partial B_{R}} \sum_{j \neq 1} u_{j} \leq C\left[D\left(\sum_{j \neq 1} u_{j}, R\right)\right]^{1 / 2}
$$

Hence

$$
\theta_{R}^{4} \leq J_{R}\left(u_{1}, \sum_{j \neq 1} u_{j}\right) \leq J_{1 / 2} \leq C\|u\|_{L^{2}\left(B_{1 / 2}\right)}^{2}\left\|\sum_{j \neq 1} u_{j}\right\|_{L^{2}\left(B_{1 / 2}\right)}^{2} .
$$

Now part (b) follows from subharmonicity. For $y$ in $B_{R}\left(x_{0}\right)$,

$$
u_{1}(y) \leq f_{B_{R}(y)} u_{1} \leq C f_{B_{2 R}\left(x_{0}\right)} u_{1} \leq 2 R\|u\|_{L^{2}\left(B_{1 / 2}\left(x_{0}\right)\right)} .
$$


Corollary 8. $u_{1}$ is Lipschitz in $B_{1 / 4}\left(x_{0}\right)$ and

$$
\left\|u_{1}\right\|_{\operatorname{Lip}\left(B_{1 / 4}\right)} \leq C\|u\|_{L^{2}\left(B_{1 / 2}\right)} .
$$

Proof. Let $y \in B_{1 / 4} \cap \operatorname{supp} u_{1}$ and $d\left(y, \partial \operatorname{supp} u_{1}\right)=h(h<1 / 4)$. Then, in $B_{h}(y)$, $u_{1}$ is positive, harmonic and $\sup \left(u_{j}\right) \leq C h$. Therefore $\left|\nabla u_{1}(y)\right| \leq \frac{C}{h} h=C$.

\section{Geometric description of the interface}

In this section we start to analyze the geometric properties of the free boundary. First, a simple

Lemma 9. If $\sum_{j>2} u_{j} \equiv 0$ in $B_{\rho}\left(x_{0}\right)$, then $u_{1}-u_{2}$ is harmonic.

Proof. $u_{1}-\sum_{j \geq 2} u_{j}$ and $u_{2}-\sum_{j \neq 2} u_{j}$ are superharmonic.

This is not a very interesting result, since it is not clear when this hypothesis holds.

To reach a reasonable description of the interface, we will complement it with two lemmas:

(a) a "clean-up" lemma that asserts that if in $B_{\rho}$ the "density" of the components "of $u_{j}$ " is very small, for $j \neq 1,2$, then $\sum_{j \neq 1,2} u_{j} \equiv 0$ in $B_{\rho / 2}$,

(b) "Almgren" monotonicity formula that says that in the complementary situation $\vec{u}$ has a tangent "cone" of homogeneity strictly bigger than one.

We start with the clean-up lemma. It consists of two parts.

The first part, a consequence of the monotonicity formula, says that if one of the components, $u_{1}$, goes to zero at a point $x_{0}$ in a "nondegenerate" fashion, i.e.,

$$
\frac{1}{r} f_{B_{r}\left(x_{0}\right)} u_{1} \geq \theta>0 \quad \text { as } r \text { goes to zero, }
$$

the whole configuration is a "small perturbation" of a linear function.

Lemma 10 (see $[\mathrm{CSa}]$ ). Assume that at $x_{0}$,

$$
D\left(u_{1}, u_{2}, 0\right)=\lim _{R \rightarrow 0} D\left(u_{1}, u_{2}, R\right)=\alpha_{0}>0 .
$$

Then:

(a) any convergent sequence of dilations $\left(1 / \lambda_{k}\right) u\left(\lambda_{k} x\right)$ for $\lambda_{k} \rightarrow 0$ converges to

$$
\bar{u}_{1}=\alpha_{1} x_{1}^{+}, \quad \bar{u}_{2}=\alpha_{2} x_{1}^{-}, \quad \bar{u}_{j} \equiv 0 \quad \text { for } j>2,
$$

(b) $\bar{u}_{1}-\bar{u}_{2}$ must be harmonic, so $\alpha_{1}=\alpha_{2}=\alpha_{0}^{1 / 4}$.

Proof. Property (a) is proven in [CSa]; note that $\left(1 / \lambda_{k}\right) u_{j}\left(\lambda_{k} x\right)$ is Lipschitz and supported in narrower and narrower domains, so $\bar{u}_{j} \equiv 0$.

(b) follows from the fact that $u_{i}-\sum_{j \neq i} u_{j}$ is superharmonic.

In these circumstances, the clean-up lemma says that the components $u_{j}$ for $j \neq 1,2$ disappear before reaching $x_{0}$. 
Theorem 11. Assume the hypotheses of the previous lemma. Then $\sum_{j>2} u_{j} \equiv 0$ in a neighborhood of $x_{0}$.

Before going into the proof, we need some preliminaries. After a large dilation, we can start with a configuration satisfying the following hypothesis.

Let $\bar{u}_{i}, i=1,2$, be the $\lambda$-dilation of $u_{i}$ at the origin, i.e. $\bar{u}_{i}(x)=u_{i}(\lambda x) / \lambda$, and write

$$
\bar{u}_{1}-\bar{u}_{2}=v_{0}+\int G(x, y) \Delta\left(\bar{u}_{1}-\bar{u}_{2}\right)=v_{0}+v_{1}
$$

where $v_{0}$ is harmonic, $\left.v_{0}\right|_{\partial B_{1}}=\bar{u}_{1}-\bar{u}_{2}$, and $v_{1}$ is the part that comes from the presence of $u_{j}, j \neq 1,2$, and is supposed to be small.

From the previous lemma, we may renormalize $\alpha_{0}=1$, and assume that

$$
\left|\left(\bar{u}_{1}-\bar{u}_{2}\right)-x_{1}\right| \leq h,
$$

in particular $\operatorname{supp}_{j \neq 1,2} \bar{u}_{j} \subset\left|\left\{\left|x_{1}\right| \leq h\right\}\right|$, and each $\bar{u}_{j}$ has Lipschitz norm less than ch.

We also recall a decay property of harmonic functions in narrow domains.

Lemma 12. Let $w$ be continuous in $B_{1}$, supported in $\Omega$ and harmonic in its support. Assume that $\Omega$ is "narrow" in the sense that any ball of radius $h, B_{h}(y)$, contained in $B_{1}$, intersects the complement of $\Omega, \mathcal{C} \Omega$, say, half of the time, i.e.,

$$
\frac{\left|B_{h} \cap \mathcal{C} \Omega\right|}{\left|B_{h}\right|}>\frac{1}{2}
$$

Then

$$
w(x) \leq \sup _{\partial B_{1}} w \cdot e^{-C(1-|x|) / h} .
$$

Proof. We prove that in the ball $B_{1-k h}, k=1, \ldots, N$, where $N \sim h^{-1}$,

$$
w(x) \leq \frac{1}{2} \sup _{B_{1-(k-1) h}} w .
$$

Indeed, by the mean value theorem,

$$
w(x) \leq f_{B_{h}(x)} w .
$$

But $w \equiv 0$ "half of the time in such a ball." Hence the estimate follows.

Before going back to the proof of the theorem, we slightly transform (3.1) into a convenient inductive hypothesis. Mainly, we change the $x_{1}$ direction to the harmonic replacement $v_{0}$ of $u_{1}-u_{2}$ in $B_{1}$, i.e., $v_{0}$ is harmonic and

$$
\left.v_{0}\right|_{\partial B_{1}}=u_{1}-u_{2} \text {. }
$$

Since $v_{0}-x_{1}$ is harmonic in $B_{1}$ and $\left.\left(v_{0}-x_{1}\right)\right|_{\partial B_{1}}=h$, we have $\left|v_{0}-\left(u_{1}-u_{2}\right)\right| \leq 2 h$ and in $B_{1 / 2},\left|\nabla\left(v_{0}-x_{1}\right)\right|=\left|\left(\nabla v_{0}\right)-e_{1}\right| \leq c h$.

Therefore, for a small number $h$, to be chosen, we have the starting hypothesis: 
Decompose $u_{1}-u_{2}=v_{0}+v_{1}$, with $v_{0}$ the harmonic replacement of $u_{1}-u_{2}$ in $B_{1}$; then:

(a) $\left|v_{0}-\left(u_{1}-u_{2}\right)\right| \leq h$,

(b) $\left|\nabla v_{0}-e\right| \leq h$,

(c) $\operatorname{supp} u_{j}$ for $j \geq 2$ is contained in the $h$-neighborhood of the Lipschitz level surface $v_{0}=0$.

Note also that $\sum_{j \neq 1,2}\left(\sup _{B_{1}} u_{j}-u_{j}\right)=\beta$ provides a barrier for $v_{1}$ since $\beta \geq 0$ and $\Delta \beta \leq \Delta\left(u_{1}-u_{2}\right)$. Hence $-\beta \leq v_{1} \leq \beta \leq C h$.

Let us see now what kind of improvement we can gain by going from $B_{1}$ to $B_{1-s}$. We note that $u_{j}$ has decreased from $h=h_{0}$ to $h_{1}=h e^{-C s / h} \leq h_{0}^{2}$ whenever $s \sim h^{1 / 2} / 2$.

In particular, if we decompose $u_{1}-u_{2}=\tilde{v}_{0}+\tilde{v}_{1}$, then $\tilde{v}_{1} \leq h_{0}^{2}$ in $B_{1-s}$. Therefore

$$
\left|u_{1}-u_{2}-\tilde{v}_{0}\right| \leq h_{0}^{2}
$$

while $\left|v_{0}-\tilde{v}_{0}\right| \leq\left|v_{0}-u_{1}-u_{2}\right| \leq h_{0}$.

To see how $v_{1}$ decays, we first estimate the total mass of the measure $\Delta u_{j}$, $j \neq 1,2$, in $B_{1-s}$. If $B_{2 \rho} \subset B_{1}$ then

$$
f_{B_{\rho}} \Delta u_{j} \leq \frac{C}{\rho^{2}} f_{B_{2 \rho}} u_{j}, \quad j \neq 1,2,
$$

implying

$$
\mu_{B_{\rho}}\left(\Delta u_{j}\right) \leq \frac{C}{\rho^{2}} \underset{B_{2 \rho}}{\operatorname{Osc}} u_{j}\left|B_{\rho}\right| .
$$

Choosing a family of balls $B_{k}=B_{\rho_{k}}, \rho_{k} \leq h^{1 / 2}$, which covers supp $u_{j}$ and using exponential decay we conclude that $\mu_{B_{1-s}} \leq \sum_{k} \rho_{k}^{-2} \operatorname{osc}_{B_{2 \rho_{k}}} u_{j}\left|B_{\rho_{k}}\right| \leq h^{2 n-1}\left|B_{1-s}\right|$, provided we take $\operatorname{osc}_{B_{2} \rho} u_{j} \leq h^{2 n}$. Now for $x \in B_{1} \operatorname{with} \operatorname{dist}\left(x, \operatorname{supp} u_{j}\right) \geq h^{1 / 2 n}$ we see from Green's representation formula that

$$
\tilde{v}_{1}(x) \leq h^{2 n-1} .
$$

Thus on $B_{1-2 s}$ we have the estimate $\left|\nabla \tilde{v}_{0}-\nabla v_{0}\right| \leq h_{0}^{1 / 2}$.

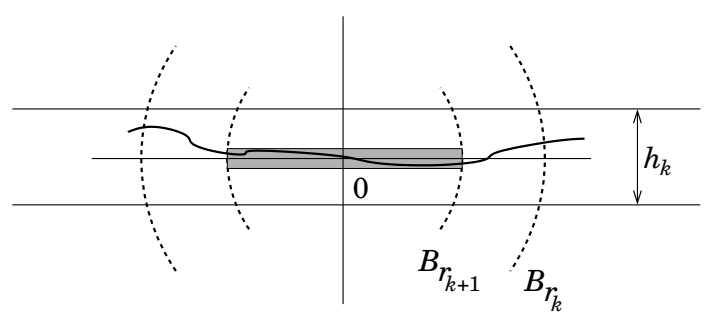

This suggests the following iterative scheme: Start with $h_{0}$ small. Consider the inductive sequence $h_{k}=h_{k-1}^{2}$ (that converges to zero very fast) and the sequence $r_{k}$ with

$$
r_{1}=1, \quad r_{k+1}=r_{k}-h_{k}^{1 / 2},
$$


which converges to $1-\mu$ with $\mu \leq 1 / 2$ if $h_{0}$ is small. Then:

Lemma 13. In $B_{r_{k}}$ there is a harmonic function $v_{k}$ such that

(a) $\left|v_{k}-\left(u_{1}-u_{2}\right)\right| \leq h_{k}$,

(b) $\left|\nabla\left(v_{k}-v_{k-1}\right)\right| \leq h_{k-1}^{2}$,

(c) $\left|\nabla v_{k}-e_{1}\right| \leq \sum_{l=0}^{k-1} h_{l}^{1 / 2} \leq 1 / 4$,

(d) the level surface $v_{k}=0$ is Lipschitz with Lipschitz constant less than 1 for every $k$.

The proof is exactly the discussion above.

Note that we take as $v_{k}$ the harmonic replacement of $u_{1}-u_{2}$ half way between $r_{k}$ and $r_{k+1}$, so it does not coincide with $u_{1}-u_{2}$ on $\partial B_{r_{k+1}}$, but still satisfies (a), and this allows us to establish the estimate (b).

\section{Almgren monotonicity formula and control of the singular set}

We will now prove, at the points of the interfaces, a monotonicity formula due to Almgren that shows that at each such point $\vec{u}$ is asymptotically homogeneous and bounds this homogeneity from below.

First we note that

Lemma 14. $(\nabla u)^{2}$ is a continuous function across the interface.

Proof. If $J_{0}\left(x_{0}\right)=\lim _{R \rightarrow 0^{+}} J_{R}\left(x_{0}\right) \neq 0$, then according to the clean-up lemma, $u_{1}-u_{2}$ is harmonic.

If $J_{0}\left(x_{0}\right)=0$ for every pair, then $|\nabla u(x)|^{2}$ goes to zero as $x$ goes to $x_{0}$ Indeed, from semicontinuity, given $\varepsilon>0$, there exist $\delta$ and $\tau$ such that

$$
J_{\delta}(x) \leq \varepsilon \quad \text { for } x \in B_{\tau}\left(x_{0}\right) .
$$

If $y \in B_{\tau / 2}\left(x_{0}\right) \cap\left\{u_{1}>0\right\}$ and $B_{s}(y)$ is the largest ball around $y$ contained in $\left\{u_{1}>0\right\}(s<\tau / 2)$, then there is a point $x_{1} \in \partial B_{s}(y) \cap\left\{u_{1}=0\right\}$. From earlier discussions, we have

$$
\frac{1}{2 s} f_{B_{2 s}\left(x_{1}\right)} u_{1}^{2} \leq \varepsilon^{1 / 2}
$$

and $\left|\nabla u_{1}(y)\right| \leq \varepsilon^{1 / 2}$.

We can now prove Almgren's monotonicity theorem [A] adapted to our setting.

Theorem 15. For $x_{0}$ in the interface define

$$
F\left(u, R, x_{0}\right)=\frac{R \int_{B_{R}\left(x_{0}\right)}|\nabla u|^{2}}{\int_{\partial B_{R}\left(x_{0}\right)} u^{2}} .
$$

Then $F^{\prime}(R) \geq 0$. 
Proof. By scale invariance it suffices to show that $(\log F)^{\prime} \geq 0$ for $R=1$. We have

$$
(\log F)^{\prime}(1)=1+\frac{\int_{\partial B_{1}}|\nabla u|^{2}}{\int_{B_{1}}|\nabla u|^{2}}-\frac{(n-1) \int_{\partial B_{1}} u^{2}+2 \int_{\partial B_{1}} u u_{r}}{\int_{\partial B_{1}} u^{2}} .
$$

Assume for the moment that $\Delta \frac{u^{2}}{2}=(\nabla u)^{2}$ as measures. Then

$$
\int_{B_{1}}|\nabla u|^{2}=\int_{B_{1}} \Delta \frac{u^{2}}{2}=\int_{\partial B_{1}} u u_{\nu}
$$

Since $u^{2}$ is subharmonic, $\Delta u^{2}$ is a positive measure, and the identity is correct except on the interface. At a regular point of the interface, where $\nabla u \neq 0$, this is also true. So we need to prove that $\Delta u^{2}$ is absolutely continuous with respect to the Lebesgue measure and that it vanishes in the Lebesgue sense at every point where $|\nabla u|^{2}=0$ and $u=0$.

At those points $x_{0}$ where $|\nabla u|$ goes to zero, $u^{2}(x) \leq o\left(\left|x-x_{0}\right|^{2}\right)$, and we have

$$
f \Delta u=o(1) \text {. }
$$

We go on with the formal computation:

$$
(\log F)^{\prime}=-(n-2)+\frac{\int_{\partial B_{1}}|\nabla u|^{2}}{\int_{\partial B_{1}} u u_{r}}-\frac{2 \int_{\partial B_{1}} u u_{r}}{\int_{\partial B_{1}} u^{2}} .
$$

We need to transform $\int_{\partial B_{1}}(\nabla u)^{2}$ into integrals involving $u$ and $u_{r}$.

We use the following Rellich identity (see [GL]):

$$
\operatorname{div}\left(x|\nabla u|^{2}\right)=n|\nabla u|^{2}+2 x_{i} u_{j} u_{i j}
$$

and

$$
\operatorname{div}\langle x, \nabla u\rangle \nabla u=|\nabla u|^{2}+\langle x, \nabla u\rangle \Delta u+x_{i} u_{j} u_{i j}
$$

or

$$
\operatorname{div}\left(x|\nabla u|^{2}-2\langle x, \nabla u\rangle \nabla u\right)=(n-2)|\nabla u|^{2}-2\langle x, \nabla u\rangle \Delta u .
$$

We now integrate (assuming that $2\langle x, \nabla u\rangle \Delta u=0$ ):

$$
\int_{B_{1}}(n-2)|\nabla u|^{2}=\int_{B_{1}} \operatorname{div}\left(x|\nabla u|^{2}-2\langle x, \nabla u\rangle \nabla u\right)=\int_{\partial B_{1}}\left[|\nabla u|^{2}-2 u_{\nu}^{2}\right],
$$

or

$$
\int_{\partial B_{1}}|\nabla u|^{2}=2 \int_{\partial B_{1}} u_{\nu}^{2}+\int_{B_{1}}(n-2)|\nabla u|^{2} .
$$

Substituting yields

$$
(\log F)^{\prime}=2\left[\frac{\int_{\partial B_{1}} u^{2}}{\int_{\partial B_{1}} u u_{r}}-\frac{\int_{\partial B_{1}} u u_{r}}{\int_{\partial B_{1}} u^{2}}\right] \geq 0 .
$$

To complete the proof we have to make sense of

$$
\int_{B_{1}}\langle x, \nabla u\rangle \Delta u=0 .
$$


We start by separating $B_{1}$ into two parts: the first one is $S_{\varepsilon}$, the $\varepsilon$-neighborhood of $S=\{x: \vec{u}(x)=0\}$, and the second one is $G_{\varepsilon}=B_{1} \backslash S_{\varepsilon}$.

Next we truncate each one of the $u_{j}$ by taking $u_{j}^{\delta}=\left(u_{j}-\delta\right)^{+}$. Each $u_{j}^{\delta}$ now has separated support:

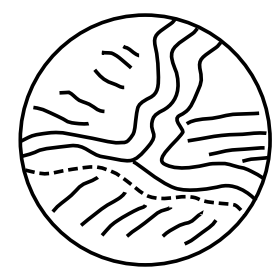

and we apply the previous calculation in each domain $D_{j}^{\delta}$ which is the interior of $\operatorname{supp} u_{j}^{\delta}$. Then we are left with the extra boundary term

$$
\int\langle x \cdot \nu\rangle|\nabla u|^{2} d A-\int 2\langle x, \nabla u\rangle\langle\nabla u, \nu\rangle d A
$$

along the analytic surfaces $u_{j}=\delta$. Since these are level surfaces of $u_{i}$, we have $\left\langle u_{i}, \nu\right\rangle=-\left|\nabla u_{i}\right|$, and also $\left|\nabla u_{i}\right| d A=d \mu_{i \delta}$ where $\mu_{i \delta}$ is the primitive measure $\Delta u_{i}^{\delta}$. The integrals above are then equal to

$$
\int\langle x, \nu\rangle\left|\nabla u_{i}\right| d \mu_{i \delta}+\int 2\langle x, \nabla u\rangle d \mu_{i \delta}=3 \int\left\langle x, \nabla u_{i}\right\rangle d \mu_{i \delta}
$$

For $\varepsilon$ fixed we now let $\delta$ go to zero.

Outside of $S_{\varepsilon}$, we have a sequence of smooth level surfaces and the integrals cancel in the limit. Inside $S_{\varepsilon},\left|\nabla u_{i}\right|=o(1)$ and therefore the integrals inside $S_{\varepsilon}$ are all bounded by

$$
\text { (total mass of } \left.\mu_{j}\right) \cdot o(1) \text {. }
$$

We then let $\varepsilon$ go to zero and the proof is complete.

\section{The singular set}

At this point, we have verified all the hypotheses necessary to develop the interface regularity theory, as in [CL3]. Therefore, we obtain the same final theorem (Theorem 4.7 there):

Theorem 16. The set of interfaces $S=\{x: \vec{u}(x)=0\}$ consists of two parts:

(a) a singular set, $\Sigma=\left\{\left|\nabla u_{j}\right|^{2}=0\right\}$, of Hausdorff dimension $n-2$,

(b) a family of analytic surfaces, level surfaces of harmonic functions.

(Note that in our case, the proof of part (b) immediately follows from the clean-up lemma.) 


\section{A final remark on the regularity of the $\varepsilon$-system}

From the Lipschitz continuity of the limiting solutions we can deduce the following regularity theorem.

Theorem 17. Let $\vec{u}^{\varepsilon}(x)=\left(u_{1}^{\varepsilon}(x), \ldots, u_{k}^{\varepsilon}(x)\right)$ be a solution of our $\varepsilon$-problem in $B_{1}$ of $\mathbb{R}^{n}$, such that

$$
\|u\| \leq 1
$$

Then, for any $\alpha<1$ and any $1<p<\infty$, $u$ is in $C^{\alpha}\left(B_{1 / 2}\right)$ and $W^{1, p}\left(B_{1 / 2}\right)$ with

$$
\|u\|_{C^{\alpha}} \leq C(\alpha), \quad\|u\|_{W^{1, p}} \leq C(p)
$$

independently of $\varepsilon$.

Proof. The proof follows from the techniques described in [CP], using the following approximation lemma.

Lemma 18. Given $\delta$, there exists $\varepsilon_{0}>0$ so that if $\varepsilon \leq \varepsilon_{0}$, and $u^{\varepsilon}$ is a solution as in Theorem 17 above, there exists a solution $u$ of the limiting problem that satisfies

$$
\left\|u_{\varepsilon}-u\right\|_{L^{\infty}\left(B_{3 / 4}\right)} \leq \delta \quad\left\|\nabla\left(u_{\varepsilon}-u\right)\right\|_{L^{2}\left(B_{3 / 4}\right)} \leq \delta .
$$

Proof. The first bound follows from equicontinuity and compactness. For the $L^{2}$ norm estimate we first point out that the total mass

$$
\sum_{i} \int_{B_{3 / 4}} \Delta u_{i}^{\varepsilon}
$$

and

$$
\int_{B_{3 / 4}}\left(\nabla u^{\varepsilon}\right)^{2}
$$

are uniformly bounded since

$$
\int_{B_{3 / 4}} \Delta u_{i}^{\varepsilon} \leq C \int_{B_{1}} u_{i}^{\varepsilon}
$$

and the gradient bound follows from Caccioppoli's inequality.

Next, notice that

$$
\Delta u^{2}=2\left(u \Delta u+(\nabla u)^{2}\right) .
$$

Then for a cut-off function $\varphi$ we write

$$
\int_{B_{1}} \varphi\left|\nabla\left(u^{\varepsilon}-u\right)\right|^{2}=-\int \varphi\left(u-u^{\varepsilon}\right) \Delta\left(u-u^{\varepsilon}\right)+\int \varphi \Delta \frac{\left(u-u^{\varepsilon}\right)^{2}}{2} .
$$

The first integral on the right-hand side goes to zero since $u-u^{\varepsilon}$ goes to zero uniformly. The second integral, after integration by parts, takes the form

$$
\int \Delta \varphi \frac{\left(u-u^{\varepsilon}\right)^{2}}{2},
$$

which goes to zero. 


\section{The parabolic case}

We will now extend our results to the evolution system

$$
\begin{cases}\Delta u_{i}^{\varepsilon}-\left(u_{i}^{\varepsilon}\right)_{t}=\frac{u_{i}^{\varepsilon}}{\varepsilon} \sum_{j \neq i} u_{j}^{\varepsilon} & \text { in } \Omega \times(-T, 0), \\ u_{i}^{\varepsilon}(x, 0)=f_{i}(x) & \text { for } x \in \Omega, \\ u_{i}^{\varepsilon}(x, t)=h_{i}(x, t) & \text { on } \partial \Omega \times(-T, 0),\end{cases}
$$

with $T>0$. It models a problem from population dynamics: the configuration of competing species which cannot coexist on the same region (competition rate is $\infty)$. We assume that $\partial \Omega$, the initial and boundary data are sufficiently smooth so that for every $\varepsilon>0$ we have a smooth solution.

More generally one can consider the Fisher equation: logistic growth equation supplemented by an extra diffusion term $\Delta$,

$$
\Delta u_{i}^{\varepsilon}(x, t)-\left(u_{i}^{\varepsilon}(x, t)\right)_{t}=\frac{1}{\varepsilon} u_{i}^{\varepsilon}(x, t) \sum_{j \neq i} u_{j}^{\varepsilon}(x, t)+g_{i}\left(x, t, u_{\varepsilon}^{i}\right)
$$

where $\Delta u_{i}^{\varepsilon}$ is the spatial diffusion, $\left(u_{i}^{\varepsilon}(t, x)\right)_{t}$ is the instantaneous rate of change of the $i$ th population's density, $\frac{1}{\varepsilon} u_{i}^{\varepsilon}(t, x) \sum_{j \neq i} u_{j}^{\varepsilon}(t, x)$ describes the interaction between different species with competition rate $1 / \varepsilon$, and $g_{i}\left(t, x, u_{\varepsilon}^{i}\right)$ is the growth rate.

As the competition rate $1 / \varepsilon$ becomes larger and larger the populations undergo a segregation and this process leads to a final configuration where the populations are separated.

As we pointed out above, the Hölder regularity theory for the elliptic $\varepsilon$-system extends to the parabolic case.

Lemma 19. If $\|u\|_{L^{\infty}\left(B_{1}\right)} \leq 1$, then $\left.u\right|_{B_{1 / 2}} \in C^{\alpha}$ and $\|u\|_{C^{\alpha}\left(B_{1 / 2}\right)} \leq C$ with $\alpha$ and $C$ independent of $\varepsilon$.

As before we will consider limits $u$ of a convergent sequence of solutions $u^{\varepsilon}$ as $\varepsilon$ goes to zero. We start with the Lipschitz regularity of the limit function $u$.

\section{Lipschitz regularity}

Since we have a uniform Hölder estimate for $u^{\varepsilon}$ the limit function $u$ is also Hölder continuous. Following the elliptic theory we start by proving the following:

Lemma 20. Let $u=\left(u_{1}, \ldots, u_{m}\right)$ be the limit function as $\varepsilon \rightarrow 0$. Then $u_{i}$ and $u_{j}$ have disjoint supports $(i \neq j)$ and $H\left(u_{i}\right) \equiv \Delta u_{i}-D_{t} u_{i}=0$ on the interior of the support of $u_{i}$.

Proof. Note that

$$
H\left(u_{i}^{\varepsilon}\right)=\Delta u_{i}^{\varepsilon}-D_{t} u_{i}^{\varepsilon}=\frac{1}{\varepsilon} u_{i}^{\varepsilon} \sum_{k \neq i} u_{k}^{\varepsilon}
$$

Then

$$
H\left(u_{1}^{\varepsilon}\right) \leq \sum_{k \neq 1} H\left(u_{k}^{\varepsilon}\right)
$$


Indeed,

$$
\begin{aligned}
\sum_{k \neq 1} H\left(u_{k}^{\varepsilon}\right) & =\frac{u_{2}^{\varepsilon}}{\varepsilon} \sum_{i \neq 2} u_{i}^{\varepsilon}+\frac{u_{3}^{\varepsilon}}{\varepsilon} \sum_{i \neq 3} u_{i}^{\varepsilon}+\cdots+\frac{u_{m}^{\varepsilon}}{\varepsilon} \sum_{i \neq m} u_{i}^{\varepsilon} \\
& =\frac{u_{1}^{\varepsilon}}{\varepsilon} \sum_{k \neq 1} u_{k}^{\varepsilon}+\text { positive terms } \geq H\left(u_{1}^{\varepsilon}\right) .
\end{aligned}
$$

Now let us assume that $\left(x_{0}, t_{0}\right) \in Q=\Omega \times(-T, 0)$ and $u_{1}^{\varepsilon}\left(x_{0}, t_{0}\right)=\alpha_{0}>0$. From Hölder continuity of $u_{1}^{\varepsilon}$ we conclude that

$$
u_{1}^{\varepsilon}(x, t) \geq \frac{\alpha_{0}}{2} \quad \text { in } Q_{h}\left(x_{0}, t_{0}\right)
$$

with $Q_{h}\left(x_{0}, t_{0}\right)=B_{h}\left(x_{0}\right) \times\left(t_{0}-h^{2} / 2, t_{0}+h^{2} / 2\right)$ for some small $h>0$. Let $\varphi(x)$ be the standard cut-off function for $B_{2 h}\left(x_{0}\right), \varphi \equiv 1$ in $B_{h}\left(x_{0}\right)$. Then in $Q_{h}=Q_{h}\left(x_{0}, t_{0}\right)$,

$$
\begin{aligned}
& \iint_{Q_{h}} H\left(u_{1}^{\varepsilon}\right) \leq \iint_{Q_{2 h}} \varphi(x) H\left(u_{1}^{\varepsilon}\right) \\
& \quad=\iint_{Q_{2 h}} \Delta \varphi(x) u_{1}^{\varepsilon}(x, t)+\int_{B_{2 h}\left(x_{0}\right)} \varphi(x)\left[u_{1}^{\varepsilon}\left(x, t_{0}+2 h^{2}\right)-u_{1}^{\varepsilon}\left(x, t_{0}-2 h^{2}\right)\right] \leq C(h) .
\end{aligned}
$$

On the other hand, $H\left(u_{1}^{\varepsilon}\right)=\frac{u_{1}^{\varepsilon}}{\varepsilon} \sum_{k \neq 1} u_{k}^{\varepsilon}$, and we have

$$
\iint_{Q_{h}} H\left(u_{1}^{\varepsilon}\right) d x d t \leq C(h) \quad \text { and } \quad \iint_{Q_{h}} \frac{u_{1}^{\varepsilon}}{\varepsilon} \sum_{k \neq 1} u_{k}^{\varepsilon} \geq \frac{\alpha_{0}}{2 \varepsilon} \iint_{Q_{h}} \sum_{k \neq 1} u_{k}^{\varepsilon} .
$$

So we conclude that

$$
\iint_{Q_{h}\left(x_{0}, t_{0}\right)} \sum_{k \neq 1} u_{k}^{\varepsilon} d x d t \leq \frac{2}{\alpha_{0}} C(h) \varepsilon .
$$

Since $u_{k}^{\varepsilon}$ 's are subsolutions, this implies that $\sum_{k \neq 1} u_{k}^{\varepsilon} \rightarrow 0$ uniformly in $Q_{h / 2}$.

To prove that $u_{i}$ is caloric in the interior of its support we use our observation

$$
H\left(u_{1}^{\varepsilon}\right) \leq \sum_{k \neq 1} H\left(u_{k}^{\varepsilon}\right)
$$

Therefore

$$
\begin{aligned}
& \iint_{Q_{h / 2}\left(x_{0}, t_{0}\right)} H\left(u_{1}^{\varepsilon}\right) \leq \iint_{Q_{h / 2}\left(x_{0}, t_{0}\right)} \sum_{k \neq 1} H\left(u_{k}^{\varepsilon}\right) \\
& \leq\left(\sum_{k \neq 1} \iint_{Q_{h}\left(x_{0}, t_{0}\right)} \Delta \eta u_{k}^{\varepsilon}(x, t)+\int_{B_{h}\left(x_{0}\right)} \eta(x)\left[u_{k}^{\varepsilon}\left(x, t_{0}+\frac{h^{2}}{2}\right)-u_{k}^{\varepsilon}\left(x, t_{0}-\frac{h^{2}}{2}\right)\right]\right) \\
& \leq C(h) \varepsilon .
\end{aligned}
$$

Here $\eta(x)$ is the standard cut-off function for $B_{h}\left(x_{0}\right)$. 
Now we are ready to prove the Lipschitz regularity. We use a parabolic version of the monotonicity formula [CSa].

Theorem 21. Let $u=\left(u_{1}, \ldots, u_{m}\right)$ be a solution in $Q_{1}$. Then

$$
\|u\|_{\operatorname{Lip}\left(Q_{1 / 2}\right)} \leq\|u\|_{L^{2}\left(Q_{1}\right)} \text {. }
$$

Proof. Recall the monotonicity formula for a pair of disjoint nonnegative subcaloric functions [CSa]. Let $u_{1}, u_{2}$ satisfy

(a) $\Delta u_{i}-D_{t} u_{i} \geq 0, i=1,2$,

(b) $u_{1} u_{2} \equiv 0$,

(c) $u_{1}(0,0)=u_{2}(0,0)=0$.

Let $\varphi(x)$ be a cut-off function such that $\varphi \equiv 0$ outside $B_{2 / 3}$ and $\varphi \equiv 1$ in $B_{1 / 2}$. Define

$$
\begin{aligned}
J(t) & =J\left(w_{1}, w_{2}, t\right) \\
& =\frac{1}{t^{2}}\left(\int_{\mathbb{R}^{n}} \int_{-t}^{0}\left|\nabla w_{1}\right|^{2} G(x,-s) d x d s \times \int_{\mathbb{R}^{n}} \int_{-t}^{0}\left|\nabla w_{2}\right|^{2} G(x,-s) d x d s\right)
\end{aligned}
$$

where $G(x, t)=t^{-n / 2} e^{-|x|^{2} / 4 t}$ and $w_{i}=u_{i} \varphi$. Then

$$
J\left(0^{+}\right)-J(t) \leq A e^{-c / t}\left\|u_{1}\right\|_{L^{2}\left(Q_{1}\right)}^{2}\left\|u_{2}\right\|_{L^{2}\left(Q_{1}\right)} .
$$

We divide the proof of the Lipschitz continuity into several steps. We start by observing that in all the estimates below there are underlying Lipschitz homogeneities.

In the first step we show that $J(t)$ controls the (weighted) product of the $L^{2}$ norm of $w_{i}$ in some strip.

Next we show that, due to the inequality $H u_{1} \leq \sum_{j \neq 1} H u_{j}$, the $w_{2}$ factor controls the $w_{1}$ factor implying its boundedness at every scale. Finally, we show that this implies spatial Lipschitz continuity for $u_{1}$.

Step 1. We prove an $L^{2}$ bound on $w_{i}$. Let $w(x, t)=u(x, t) \varphi(x)$. Then, by direct computation,

$$
H\left(w^{2}\right)=\Delta\left(w^{2}\right)-D_{t} w^{2}=2 w \Delta w+2|\nabla w|^{2}-2 w w_{t} .
$$

Hence

$|\nabla w|^{2}=\frac{1}{2} H\left(w^{2}\right)-w \Delta w+w w_{t}=\frac{1}{2} H\left(w^{2}\right)-w[\Delta u \varphi+2 \nabla u \nabla \varphi+u \Delta \varphi]+w u_{t} \varphi$.

Integrating this identity with respect to the measure $d \mu=G(x,-s) d x d s$ we get

$$
\begin{aligned}
\int_{-t}^{0} \int_{\mathbb{R}^{n}}|\nabla w|^{2} d \mu= & \int_{-t}^{0} \int_{\mathbb{R}^{n}} \frac{1}{2} H\left(w^{2}\right) d \mu-\int_{-t}^{0} \int_{\mathbb{R}^{n}} w[\Delta u \varphi+2 \nabla u \nabla \varphi+u \Delta \varphi] d \mu \\
& +\int_{-t}^{0} \int_{\mathbb{R}^{n}} w u_{s} \varphi d \mu .
\end{aligned}
$$


Note that

$$
\begin{aligned}
\int_{-t}^{0} \int_{\mathbb{R}^{n}} & \frac{1}{2} H\left(w^{2}\right) G(x,-s) d x d s \\
= & \frac{1}{2} \int_{-t}^{0} \int_{\mathbb{R}^{n}}\left[w^{2} \Delta G(x,-s)-D_{s} w^{2} G(x,-s)\right] d x d s \\
= & \frac{1}{2} \int_{-t}^{0} \int_{\mathbb{R}^{n}} w^{2}\left[\Delta G(x,-s)+D_{s} G(x,-s)\right] d x d s+\frac{1}{2} \int_{\mathbb{R}^{n}} w^{2}(x,-t) G(x, t) d x \\
= & \frac{1}{2} w^{2}(0,0)+\frac{1}{2} \int_{\mathbb{R}^{n}} w^{2}(x,-t) G(x, t) d x
\end{aligned}
$$

since $\Delta G(x,-s)+D_{s} G(x,-s)=\delta_{0,0}$.

Therefore we conclude that

$$
\begin{aligned}
\int_{-t}^{0} \int_{\mathbb{R}^{n}}|\nabla w|^{2} G(x,-s) d x d s= & \frac{1}{2} w^{2}(0,0)+\frac{1}{2} \int_{\mathbb{R}^{n}} w^{2}(x,-t) G(x, t) d x \\
& -\int_{-t}^{0} \int_{\mathbb{R}^{n}} w \varphi H(u) G(x,-s) d x d s \\
& -\int_{-t}^{0} \int_{\mathbb{R}^{n}} w[2 \nabla u \nabla \varphi+u \Delta \varphi] G(x,-s) d x d s .
\end{aligned}
$$

Now if $u=u_{1}$, then $w_{1}=u_{1} \cdot \varphi$ and

$$
\int_{-t}^{0} \int_{\mathbb{R}^{n}} w \varphi H\left(u_{1}\right) G(x,-s) d x d s=\int_{-t}^{0} \int_{\mathbb{R}^{n}} \varphi^{2} u_{1} H\left(u_{1}\right) G(x,-s) d x d s=0
$$

since $u_{1} H\left(u_{1}\right)=0$ and $w_{1}(0,0)=0$, so

$$
\begin{aligned}
I_{1}(t) & =\int_{-t}^{0} \int_{\mathbb{R}^{n}}\left|\nabla w_{1}\right|^{2} G(x,-s) d x d s \\
& =\frac{1}{2} \int_{\mathbb{R}^{n}} w_{1}^{2}(x,-t) G(x, t) d x-\int_{-t}^{0} \int_{\mathbb{R}^{n}} w_{1}\left[2 \nabla u_{1} \nabla \varphi+u_{1} \Delta \varphi\right] G(x,-s) d x d s .
\end{aligned}
$$

Observe that the last term on the right admits an estimate

$$
\begin{aligned}
& \int_{-t}^{0} \int_{\mathbb{R}^{n}} w_{1}[\left.2 \nabla u_{1} \nabla \varphi+u_{1} \Delta \varphi\right] G(x,-s) d x d s \\
& \leq C \int_{-t}^{0} \int_{B_{2 / 3} \backslash B_{1 / 2}}|w|\left(\left|\nabla u_{1}\right|+u_{1}\right) G(x,-s) d x d s \leq C e^{-c / t}
\end{aligned}
$$

where $C$ depends on the $L^{2}$ norm of $u_{1}$. Now we consider $w_{2}=\tilde{u} \varphi$, where $\tilde{u}=$ $\sum_{k \neq 1} u_{k}$. Note that $u_{1}$ and $\sum_{k \neq 1} u_{k}$ satisfy the assumption of the monotonicity formula. 
Next, for $w_{2}$ we have

$$
\begin{aligned}
I_{2}(t)= & \int_{-t}^{0} \int_{\mathbb{R}^{n}}\left|\nabla w_{2}\right|^{2} G(x,-s) d x d s \\
= & \frac{1}{2} \int_{\mathbb{R}^{n}} w_{2}^{2} G(x, t) d x-\int_{-t}^{0} \int_{\mathbb{R}^{n}} w_{2} \varphi H(\tilde{u}) G(x,-s) d x d s \\
& -\int_{-t}^{0} \int_{\mathbb{R}^{n}} w_{2}[w \nabla \tilde{u} \nabla \varphi+\tilde{u} \Delta \varphi] G(x,-s) d x d s .
\end{aligned}
$$

If at $(x, t)$ we have $u_{2}(x, t)>0$, then $H u_{2}(x, t)=0$, and since $u_{k}$ 's have disjoint supports,

$$
\tilde{u} H(\tilde{u})=0 .
$$

If $(x, t)$ is a free boundary point, then $\tilde{u}(x, t)=0$. Hence

$$
\int_{-t}^{0} \int_{\mathbb{R}^{n}} \varphi^{2} \tilde{u} H(\tilde{u}) G(x,-s) d x d s=0
$$

and as in the case of $w_{1}$,

$$
\int_{-t}^{0} \int_{\mathbb{R}^{n}} w_{2}[2 \nabla \tilde{u} \nabla \varphi+\tilde{u} \Delta \varphi] G(x,-s) d x d s \leq C e^{-c / t} .
$$

Combining the estimates for $I_{1}$ and $I_{2}$ we have

$$
\begin{aligned}
J(t)=\frac{1}{t^{2}} I_{1}(t) I_{2}(t) \geq & \frac{1}{t^{2}}\left(\frac{1}{2} \int_{\mathbb{R}^{n}} w_{1}^{2}(x,-t) G(x, t) d x+O\left(e^{-c / t}\right)\right) \\
& \times\left(\frac{1}{2} \int_{\mathbb{R}^{n}} w_{2}^{2}(x,-t) G(x, t) d x+O\left(e^{-c / t}\right)\right) .
\end{aligned}
$$

This means that

$$
\frac{1}{4 t^{2}} \int_{\mathbb{R}^{n}} w_{1}^{2}(x,-t) G(x, t) d x \int_{\mathbb{R}^{n}} w_{2}^{2}(x,-t) G(x, t) d x \leq J(t)+O\left(e^{-c / t}\right) .
$$

Step 2. Next we want to show that the $w_{1}$-term is controlled by the $w_{2}$-term. Recall that $H\left(u_{1}\right) \leq H(\tilde{u})$ so

$$
\begin{aligned}
0 \leq & \int_{-t}^{0} \int_{\mathbb{R}^{n}} H\left(\tilde{u}-u_{1}\right) \varphi G(x,-s) d x d s \\
= & \int_{-t}^{0} \int_{\mathbb{R}^{n}}\left(\tilde{u}-u_{1}\right) \Delta[\varphi G(x,-s)] d x d s-\int_{-t}^{0} \int_{\mathbb{R}^{n}}\left(\tilde{u}-u_{1}\right)_{s} \varphi G\left(x_{1}-s\right) d x d s \\
= & \int_{-t}^{0} \int_{\mathbb{R}^{n}}\left(\tilde{u}-u_{1}\right)\left[\Delta\left(\varphi G\left(x_{1}-s\right)\right)+D_{s}\left(\varphi G\left(x_{1}-s\right)\right)\right] d x d s \\
& +\int_{\mathbb{R}^{n}}\left(\tilde{u}-u_{1}\right) \varphi(x,-t) G(x, t) d x
\end{aligned}
$$




$$
\begin{aligned}
= & \int_{-t}^{0} \int_{\mathbb{R}^{n}}\left(\tilde{u}-u_{1}\right)[\Delta \varphi G(x,-s)+2 \nabla \varphi \nabla G(x,-s)+\varphi \Delta G(x,-s) \\
& \left.+\int_{\mathbb{R}^{n}}\left(w_{2}(x,-t)-w_{1}(x,-t)\right) G(x, t) d x d s \quad+\varphi(x) D_{s} G\left(x_{1}-s\right)\right] d x d s \\
= & \int_{-t}^{0} \int_{\mathbb{R}^{n}}\left(\tilde{u}-u_{1}\right)\left[\Delta \varphi+\nabla \varphi \cdot \frac{x}{t}\right] G(x,-s) d x d s \\
& +\int_{\mathbb{R}^{n}}\left(w_{2}(x,-t)-w_{1}(x,-t)\right) G(x, t) d x \\
= & O\left(e^{-c / t}\right)+\sqrt{t}\left(\theta_{2}(t)-\theta_{1}(t)\right)
\end{aligned}
$$

where

$$
\theta_{i}(t)=\frac{1}{\sqrt{t}} \int_{\mathbb{R}^{n}} w_{i}(x,-t) G(x, t) d x, \quad i=1,2 .
$$

Therefore

$$
\theta_{1}(t) \leq \theta_{2}(t)+O\left(e^{-c / t}\right) .
$$

After applying the Cauchy-Schwarz inequality we have

$$
\begin{aligned}
& \theta_{1}(t) \leq\left(\frac{1}{t} \int_{\mathbb{R}^{n}} w_{1}^{2}(x,-t) G(x, t) d x\right)^{1 / 2}, \\
& \theta_{1}(t) \leq \theta_{2}(t) \leq\left(\frac{1}{t} \int_{\mathbb{R}^{n}} w_{2}^{2}(x,-t) G(x, t) d x\right)^{1 / 2} .
\end{aligned}
$$

Multiplying both inequalities we get

$$
\theta_{1}^{4}(t) \leq \frac{1}{t^{2}} \int_{\mathbb{R}^{n}} w_{1}^{2}(x,-t) G(x, t) d x \int_{\mathbb{R}^{n}} w_{2}^{2}(x,-t) G(x, t) d x \leq 4\left(J(t)+O\left(e^{-c / t}\right)\right) .
$$

Therefore the monotonicity formula implies that $\theta_{1}(t)$ is bounded for any $t$ small.

Step 3. Since the heat equation is translation invariant, we can extend the previous estimate to any free boundary point $\left(x_{0},-t_{0}\right) \in Q$ with $t_{0}>0$. For some $\rho_{0}>0$ we have $B_{\rho_{0}}\left(x_{0}\right) \times\left(-t_{0}-\rho_{0},-t_{0}\right) \subset Q$. Here $\rho_{0}$ depends only on the distance of $\left(x_{0},-t_{0}\right)$ from the parabolic boundary of $Q$. Then we let $\eta=x-x_{0}, \tau=t+t_{0}$ and note that $v_{i}(\eta, \tau)=u_{i}\left(x_{0}+\eta, \tau-t_{0}\right)$ is also a solution. Taking $t=r^{2}$ in the definition of $\theta_{i}(t), t>0$, and using a change of variables $x=r y$ we have

$$
\frac{1}{r} \int_{\mathbb{R}^{n}} u_{1}\left(x_{0}+y r,-t_{0}-r^{2}\right) \varphi\left(x_{0}+y r\right) G(y, 1) d y \leq C_{0}
$$

for any point $\left(x_{0},-t_{0}\right)$ such that $\operatorname{dist}\left(\left(x_{0},-t_{0}\right), \partial_{p} Q\right) \geq \rho_{0}$ and $C_{0}$ depends on $\rho_{0}$.

Next we want to show that $u$ grows linearly away from the free boundary. Assume that $\left(x_{1},-t_{1}\right) \in Q, t_{1}>0, u_{1}\left(x_{1},-t_{1}\right)>0$ and let $\rho$ be the distance 
of $\left(x_{1},-t_{1}\right)$ from the free boundary. Hence $u_{1}$ is caloric in $Q_{1}=B_{\rho / 2}\left(x_{1}\right) \times$ $\left(-t_{1}-\rho^{2} / 4,-t_{1}\right)$. Suppose that for some $x_{2}$ we have

$$
u_{1}\left(x_{2},-t_{1}\right) \geq M R
$$

with $R=\rho / 2$ and $M \gg 1$.

By the Harnack inequality,

$$
\inf _{B_{R}\left(x_{1}\right) \times\left(-t_{1}-3 R^{2} / 4,-t_{0}-R^{2} / 2\right)} u_{1} \geq C_{1} \sup _{B_{R}\left(x_{1}\right) \times\left(-t_{1}-R^{2} / 4,-t_{0}\right)} u_{1} \geq C_{1} R M .
$$

Thus taking $r=4 R=2 \rho$ in (6.2) we obtain, for every $R$,

$$
C_{0} \geq \int_{\mathbb{R}^{n}} \frac{u_{1}\left(x_{0}+4 R y,-t_{1}-R^{2} / 2-(4 R)^{2}\right)}{4 R} G(y, 1) d y \geq c(n) M C_{1} .
$$

which is a contradiction if $M>C_{0} /\left(c(n) C_{1}\right)$.

Theorem 22. $u(x, t)$ is locally Lipschitz in the parabolic distance.

Proof. It is a standard argument to show that the Lipschitz continuity in space implies $\frac{1}{2}$-Hölder continuity in time.

\section{The clean-up lemma}

We start by pointing out that in a "clean" neighborhood of a free boundary point, $u_{1}-u_{2}$ is caloric.

Lemma 23. If $\sum_{j>2} u_{j} \equiv 0$ in some cylinder $Q_{\rho}\left(x_{0}, t_{0}\right)$ then $u_{1}-u_{2}$ is caloric in $Q_{\rho}\left(x_{0}, t_{0}\right)$.

Proof. Since

$$
H\left(u_{1}\right) \leq H\left(\sum_{k \neq 1} u_{k}\right)=H\left(u_{2}\right)+H\left(\sum_{k>2} u_{k}\right)
$$

and

$$
H\left(u_{2}\right) \leq H\left(\sum_{k \neq 2} u_{k}\right)=H\left(u_{1}\right)+H\left(\sum_{k>2} u_{k}\right)
$$

it follows that $u_{1}-u_{2}$ is caloric in $Q_{\rho}\left(x_{0}, t_{0}\right)$.

Next we have the parabolic clean-up lemma, which plays a crucial role in the classification of singular points of the free boundary. It basically says that if at some free boundary point $\left(x_{0}, t_{0}\right), J\left(0^{+}\right)>0$, that is, $\left|\nabla u\left(x_{0}, t_{0}\right)\right| \neq 0$, then at some neighborhood of $\left(x_{0}, t_{0}\right)$ we have exactly two phases.

Clean-Up Lemma. Assume that at $\left(x_{0}, t_{0}\right)$,

$$
J\left(0^{+}\right)=\lim _{t \rightarrow 0^{+}} J(t)=\lambda>0 .
$$

Then $\sum_{j>2} u_{j} \equiv 0$ in a neighborhood of $\left(x_{0}, t_{0}\right)$.

First recall the following result [CSa]. 
Lemma 24 (see $[\mathrm{CSa}])$. Assume that at $\left(x_{0}, t_{0}\right)$,

$$
J\left(u_{1}, u_{2}, 0\right)=\lim _{t \rightarrow 0} J\left(u_{1}, u_{2}, t\right)=\alpha_{0}>0 .
$$

Then:

(a) any convergent sequence of dilations $\left(1 / \lambda_{k}\right) u\left(\lambda_{k} x, \lambda_{k} t\right)$ for $\lambda_{k} \rightarrow 0$ converges to

$$
\bar{u}_{1}=\alpha_{1} x_{1}^{+}, \quad \bar{u}_{2}=\alpha_{2} x_{1}^{-}, \quad \bar{u}_{j} \equiv 0 \quad \text { for } j>2,
$$

(b) $\bar{u}_{1}-\bar{u}_{2}$ must satisfy the heat equation, so $\alpha_{1}=\alpha_{2}=\alpha_{0}^{1 / 4}$.

In these circumstances, the clean-up lemma says that the components $u_{j}$ for $j \neq 1,2$ decay faster than $u_{1}, u_{2}$ and vanish before reaching $\left(x_{0}, t_{0}\right)$.

Theorem 25. Let $u_{1}, u_{2}$ be as in the lemma above. Then $\sum_{j>2} u_{j} \equiv 0$ in a neighborhood of $\left(x_{0}, t_{0}\right)$.

Before going into the proof, we need some preliminaries. After a large dilation, we can start with a configuration satisfying the following hypothesis.

Let $\bar{u}_{i}, i=1,2$, be the $\lambda$-dilation of $u_{i}$ at the origin, i.e. $\bar{u}_{i}(x, t)=u_{i}(\lambda x, \lambda t) / \lambda$, and write

$$
\bar{u}_{1}-\bar{u}_{2}=v_{0}+v_{1}
$$

where $v_{0}$ is caloric, $\left.v_{0}\right|_{\partial B_{1}}=\bar{u}_{1}-\bar{u}_{2}$, and $v_{1}$ is the part that comes from the presence of $u_{j}, j \neq 1,2$, and is supposed to be small.

From the previous lemma, we may renormalize $\alpha_{0}=1$, and assume that

$$
\left|\left(\bar{u}_{1}-\bar{u}_{2}\right)-x_{1}\right| \leq h,
$$

in particular $\operatorname{supp}_{j \neq 1,2} \bar{u}_{j} \subset\left|\left\{\left|x_{1}\right| \leq h\right\}\right|$, and $\bar{u}_{j}$ being Lipschitz, $\bar{u}_{j} \leq h$.

We also recall a decay property of harmonic functions in narrow domains.

Lemma 26. Let $w$ be continuous in $\mathcal{C}_{1}=B_{1} \times[-1,1]$, supported in $\Omega \subset \mathcal{C}_{1}$ and harmonic in its support. Assume that $\Omega$ is "narrow" in the sense that any cylinder $Q_{h}=B\left(x_{0}\right) \times\left(t_{0}-h^{2}, t_{0}\right)$, contained in $\mathcal{C}_{1}$, intersects $\mathcal{C} \Omega$, say, half of the time, i.e.,

Then

$$
\frac{\left|Q_{h} \cap \mathcal{C} \Omega\right|}{\left|Q_{h}\right|}>\frac{1}{2}
$$

$$
w(x) \leq \sup _{\partial_{p} \mathcal{C}_{1}} w \cdot e^{-C\left(1-\sqrt{|x|^{2}+t}\right) / h} .
$$

Proof. We prove that in $Q_{i, k}=Q_{h}\left(x_{i},-1+2 k h\right), k=1, \ldots, N, x_{i} \in h \mathbb{Z}^{2} \cap \mathcal{C}_{1}$, where $N \sim h^{-2}$, we have

$$
w(x) \leq \frac{1}{C} \sup _{Q_{i k}} w
$$

for some $C>1$. Indeed, by a density estimate we have

$$
\sup _{Q_{i, k}} w(x, t) \leq \frac{1}{1+c_{0} \frac{\left|\mathcal{C} \Omega \cap Q_{i, k-1}\right|}{\left|Q_{i, k-1}\right|}} \sup _{Q_{i, k-1}} w .
$$


But $w \equiv 0$ "half of the time", hence $\left|\mathcal{C} \Omega \cap Q_{i, k-1}\right| /\left|Q_{i, k-1}\right| \geq 1 / 2$. Repeating this for all $i, k$ and combining the estimates yields the result.

Now we start the proof of the parabolic clean-up lemma.

Proof. From the proof of the monotonicity formula [CSa], we know that the blowup functions are a pair of linear functions, and from the $H(u)$ inequalities they have the same slope. This means that near $\left(x_{0}, t_{0}\right)$ we have uniform flatness at every scale.

As in the elliptic case we want to start with a suitable inductive hypothesis.

In fact, the iterative scheme is the same as in the elliptic case. Start with $h_{1}$ small. Consider the inductive sequence $h_{k}=h_{k-1}^{2}$ (that converges to zero very fast) and the sequence $r_{k}$ with

$$
r_{1}=1, \quad r_{k+1}=r_{k}-h_{k}^{1 / 2}
$$

which converges to $1-\mu$ with $\mu \leq 1 / 2$ if $h_{1}$ is small.

More precisely, we can state

Lemma 27. In $\mathcal{C}_{r_{k}}=B_{r_{k}} \times\left(-1+h_{k}^{1 / 2}, 1-h_{k}^{1 / 2}\right)$ there is a caloric function $v_{k}$ such that

(a) $\left|v_{k}-\left(u_{1}-u_{2}\right)\right| \leq h_{k}$,

(b) $\left|\nabla\left(v_{k}-v_{k-1}\right)\right| \leq h_{k}^{1 / 2}$,

(c) $\left|\nabla v_{k}-e_{1}\right| \leq \sum_{l=1}^{k} h_{l}^{1 / 2} \leq 1 / 4$,

(d) the level surface $v_{k}=0$ is Lipschitz with Lipschitz constant less than 1 for every $k$.

To prove this we proceed as follows. First from the exponential decay we can estimate $\tilde{v}_{0}-\left(u_{1}-u_{2}\right)$ in the cylinder $\mathcal{C}_{1-s}$. Next using the covering argument and computation from the previous section one can estimate the size of $\Delta u-u_{t}$ in $\mathcal{C}_{1-s}$ and then from Green's representation theorem we find that $v_{1}$ decays as $h^{2 n-1}$ away from the $h^{1 / 2 n}$-neighborhood of supp $u_{j}, j>2$. Finally, using gradient estimates we conclude that $\left|\nabla \tilde{v}_{0}-\nabla v_{0}\right| \leq h^{1 / 2}$.

As in the elliptic theory, we now have a discontinuity. At the neighborhood of a clean point the free boundary is a transversal level surface of a caloric function. At a singular point the gradient of $u$ goes to zero, and we want to classify such points.

\section{Almgren's formula}

Lemma 28. $(\nabla u)^{2}$ is a continuous function across the interface.

Proof. If $J_{0}\left(x_{0}, t_{0}\right)=\lim _{t \rightarrow 0^{+}} J_{t}\left(x_{0}, t_{0}\right) \neq 0$, then from the clean-up lemma $u_{1}-u_{2}$ is harmonic.

If $J_{0}\left(x_{0}, t_{0}\right)$ is zero for every pair, then $|\nabla u(x, t)|^{2}$ goes to zero as $x$ goes to $x_{0}$, which follows from the estimates of $\theta_{i}(t), i=1,2$. 
We now consider the backward heat equation

$$
\Delta u+u_{t}=0 \quad \text { in } \mathbb{R}_{+}^{n+1} .
$$

For $t_{0}>0$, we define

$$
H(t)=\int_{\mathbb{R}^{n}}|u(x, t)|^{2} G(x, t) d x
$$

where

$$
G(x, t)=\frac{1}{\left(t+t_{0}\right)^{n / 2}} e^{-\frac{|x|^{2}}{4\left(t+t_{0}\right)}} \quad \text { and } \quad u=\left(u_{1}, \ldots, u_{m}\right) \text {. }
$$

Also

$$
D(t)=\int_{\mathbb{R}^{n}}|\nabla u(x, t)|^{2} G(x, t) d x .
$$

Theorem 29 (Parabolic Almgren monotonicity formula).

$$
N(t)=\frac{\left(t+t_{0}\right) D(t)}{H(t)} \text { is decreasing. }
$$

Proof. A version of this theorem is due to [EFV] for caloric functions. For completeness we give a proof in Section 11 with the modification for our particular case.

We are now in the following situation. Our solutions are only local and it is well-known that solutions of the heat equation in $B_{1} \times(0, \infty)$ with suitable nonhomogeneous time dependent boundary data prescribed on the lateral boundary $\partial B_{1} \times(0, \infty)$ may become identically zero for $t \geq T$. We would like to prove the following: given a free boundary point, unless our solution is identically zero in a cylinder backwards in time (i.e. had already become identically zero all the way to the boundary), it is forced to have a polynomial decay at the point, so that we can "blow it up" to a nontrivial solution integrable at infinity against the Gaussian kernel.

We can ensure this by a modification to our setting of a theorem of L. Escauriaza, F. J. Fernández and S. Vessella.

Theorem 30. Let $\left(u_{1}, \ldots, u_{m}\right)$ be a solution. Then there exists a constant $C$ such that

$$
\int_{Q_{2 r}} u^{2} \leq C \int_{Q_{r}} u^{2} .
$$

This estimate is proved in [EFV] for a class of constant coefficient parabolic equations. The main part of the proof is based upon a localization of Almgren's formula by multiplying $u$ with a cut-off function. Since in our case $N(t)$ is a monotone function and all computations for derivatives of $D(t)$ and $H(t)$ remain valid, the doubling property of the solution now immediately follows from the proof of Theorem 29 and [EFV]. 


\section{Classification of the global solutions}

If $N(t)=\lambda$ for all $t$ and $\lambda>0$, then from the proof of the monotonicity formula we get

$$
u_{t}+\frac{x-x_{0}}{2\left(t+t_{0}\right)} \nabla u=c(t) u(x, t)
$$

for some unknown function $c(t)$. We want to show that $c(t)$ is the homogeneity degree of $u$. Without loss of generality we may assume that $x_{0}=0, t_{0}=0$. Then we have

$$
u_{t}(x, t)+\frac{x}{2 t} \nabla u=c(t) u(x, t) .
$$

For $\theta>0$ we consider $u_{\theta}(x, t)=u\left(x \theta, t \theta^{2}\right)$. Then

$$
\frac{d}{d \theta} u_{\theta}=u_{t}\left(x \theta_{1}+\theta^{2}\right) 2 \theta t+\nabla\left(x \theta, t \theta^{2}\right) x .
$$

$u_{\theta}$ satisfies a differential equation on the path $\left(x \theta, t \theta^{2}\right)$ for fixed $(x, t)$. Indeed,

$$
\begin{aligned}
u_{t}\left(x \theta, t \theta^{2}\right)+\frac{x \theta}{2 t \theta^{2}} \nabla u\left(x \theta, t \theta^{2}\right) & =c\left(t \theta^{2}\right) u\left(x \theta, t \theta^{2}\right), \\
2 t \theta u_{t}\left(x \theta, t \theta^{2}\right)+x \cdot \nabla u\left(x \theta, t \theta^{2}\right) & =2 t \theta c\left(t \theta^{2}\right) u_{\theta}(x, \theta),
\end{aligned}
$$

or

$$
\frac{d}{d \theta} u_{\theta}=\frac{H\left(t \theta^{2}\right)}{\theta} u_{\theta}(x, t)
$$

where $H\left(t \theta^{2}\right)=2 c\left(t \theta^{2}\right) t \theta^{2}$. Hence

$$
\left.\log u_{\theta}\right|_{1} ^{\theta}=\int_{1}^{\theta} \frac{H\left(t \sigma^{2}\right)}{\sigma} d \sigma
$$

and

$$
u_{\theta}(x, t)=e^{\int_{1}^{\theta} \frac{H\left(t \sigma^{2}\right)}{\sigma} d \sigma} u(x, t) .
$$

Since $u_{\theta}(x, t)$ satisfies the backward heat equation we get

$$
0=H\left(u_{\theta}\right)=H(u) \cdot e^{\int_{1}^{\theta} \frac{H\left(t \sigma^{2}\right)}{\sigma} d \sigma}+u(x, t) \frac{d}{d t} e^{\int_{1}^{\theta} \frac{H\left(t \sigma^{2}\right)}{\sigma} d \sigma} .
$$

Therefore $\int_{1}^{\theta} \frac{H\left(t \sigma^{2}\right)}{\sigma} d \sigma=c(\theta)$ does not depend on $t$. Differentiating this equality with respect to $t$ we get

$$
0=\int_{1}^{\theta} H^{\prime}\left(t \sigma^{2}\right) \sigma d \sigma=\frac{H\left(t \theta^{2}\right)-H(t)}{2 t},
$$

so $H$ is a constant. Recall that $H(s)=2 c(s) s$, implying that

$$
c(s)=\frac{\alpha}{2 s},
$$

where $\alpha$ is a constant, therefore $u$ satisfies

$$
u_{t}+\frac{x}{2 t} \nabla u \cdot x=\frac{\alpha}{2 t} u .
$$


Thus we conclude that

$$
u\left(x \theta, t \theta^{2}\right)=\theta^{\alpha} u(x, t), \quad u=\left(u_{1}, \ldots, u_{k}\right),
$$

that is, $u$ is homogeneous of degree $\alpha$ on the paths $\left(x \theta, t \theta^{2}\right)$.

Since $u$ is homogeneous we can seek the solution $u$ in the form $t^{\alpha / 2} f(x / \sqrt{t})$. In particular, it can be a traveling wave $u(x, t)=\left(x^{2}+c t\right)^{\alpha / 2}$. Consider

Then

$$
u(x, t)=t^{\alpha / 2} f\left(\frac{x}{\sqrt{t}}\right) .
$$

$$
\begin{aligned}
u_{t} & =\frac{\alpha}{2} t^{\alpha / 2-1} f\left(\frac{x}{\sqrt{t}}\right)+t^{\alpha / 2} \nabla f\left(\frac{x}{\sqrt{t}}\right)\left(-\frac{x}{2 t^{3 / 2}}\right), \\
u_{x} & =t^{\alpha / 2-1 / 2} f_{x}\left(\frac{x}{\sqrt{t}}\right), \\
u_{x x} & =t^{\alpha / 2-1} f_{x x}\left(\frac{x}{\sqrt{t}}\right) .
\end{aligned}
$$

Plugging these into the backward heat equation $\Delta u+u_{t}=0$ we obtain

$$
-\Delta f(z)+\frac{1}{2} \nabla f(z) \cdot z=\frac{\alpha}{2} f(z)
$$

where $z=x / \sqrt{t}$. Therefore $\alpha / 2$ is an eigenvalue of the operator $-\Delta+\frac{1}{2} \nabla \cdot z$ and $u$ is the corresponding eigenfunction.

In the one-dimensional case, $f$ satisfies an ODE

$$
2 f_{z z}-f_{z} z+\alpha f=0 .
$$

Setting $w(z)=f(2 z)$ one can easily verify that $w$ solves $w_{z z}-2 w_{z} z+2 \alpha w=0$. But the latter is the differential equation for the Hermite polynomials which can be explicitly given by

$$
w(z)=\alpha ! \sum_{k=0}^{[\alpha / 2]} \frac{(-1)^{k}(2 z)^{\alpha-2 k}}{k !(n-2 k) !} .
$$

Hence returning to $f$ we obtain

$$
\begin{aligned}
u(x, t) & =\alpha ! t^{\alpha / 2} f\left(\frac{x}{\sqrt{t}}\right)=\alpha ! t^{\alpha / 2} \sum_{k=0}^{[\alpha / 2]} \frac{(-1)^{k}}{k !(n-2 k) !}\left(\frac{x}{\sqrt{t}}\right)^{\alpha-2 k} \\
& =\alpha ! \sum_{k=0}^{[\alpha / 2]} \frac{(-1)^{k}}{k !(n-2 k) !} x^{\alpha-2 k} t^{k},
\end{aligned}
$$

which is the $\alpha$-caloric polynomial for the backward heat equation. Now if one has the heat equation (i.e. after replacing $t$ with $-t$ ) then

$$
h_{m}(x, s)=m ! \sum_{k=0}^{[m / 2]} \frac{1}{k !(n-2 k) !} x^{m-2 k} t^{k}
$$

is the solution for our problem in the one-dimensional case. 
In $n$ dimensions, $h_{m_{1}}\left(x_{1}, s\right) h_{m_{2}}\left(x_{2}, s\right) \cdots h_{m_{n}}\left(x_{n}, s\right)$ with $\sum_{j=1}^{n} m_{j}=m$, $m_{j} \geq 0$, is the homogeneous solution of degree $m$ of our problem. By the classical theory of Hermite polynomials they have only simple real zeros. Hence the polynomial $h_{m}(x,-1)$ has $m$ simple zeros. Furthermore, $h_{m}(x, s)$ is even or odd in the variable $x$ when $m$ is an even or odd integer, respectively. Therefore we can describe the nature of the nodal sets of $h_{m}$ in spacetime,

$$
\Sigma\left(h_{m}\right)=\left\{(x, s): h_{m}(x, s)=0\right\} .
$$

First notice that $h_{0}(x, s) \equiv 1$ so $\Sigma\left(h_{0}\right)=\emptyset$, while $h_{1}(x, s)=x$ and $\Sigma\left(h_{1}\right)$ is the $t$-axis. Hence $h_{m}$ has a degenerate zero if and only if $m \geq 2$.

\section{Structure of the singular set}

In this section we establish an estimate for the parabolic Hausdorff dimension of the set $\Sigma=\{(x, t): u(x, t)=0,|\nabla u(x, t)|=0\}$.

Theorem 31. Let $\mathcal{P}$ be the parabolic Hausdorff measure. Then the parabolic Hausdorff dimension $\operatorname{dim}_{\mathcal{P}} \Sigma(u)$ is at most $n$.

For the definition of $\mathcal{P}$ see [LY]. The proof is based on Federer's dimension reduction argument. We sketch it here. Let $\mathcal{F}$ be the set of all solutions and take $u \in \mathcal{F}$ and let $\mathcal{S}: \mathcal{F} \rightarrow \mathcal{C}$, where $\mathcal{S}$ is the singular map, $\mathcal{S}(u)=\Sigma$, and $\mathcal{C}$ is the collection of all closed sets in $\mathbb{R}^{n} \times \mathbb{R}$. First notice that the following hypotheses are satisfied (see [LY, p. 51]):

H1. $\mathcal{F}$ is closed under translation and scaling.

H2. Existence of homogeneous degree zero tangent functions.

H3. Singular set hypothesis, i.e. the existence of the mapping $\Sigma$.

If $\mathrm{H} 1-\mathrm{H} 2$ are satisfied then the pair $(\mathcal{F}, \mathcal{S})$ is locally asymptotically self-similar.

It is easy to see that H1 is satisfied. Next notice that from Almgren's theorem and nondegeneracy (polynomial growth from below) the scaled function $\lambda^{-N} u\left(\lambda x, \lambda^{2} t\right)$ converges to a caloric polynomial by our classification of the global profiles. Here $N$ is a positive integer. Finally, H3 is satisfied in view of the local regularity of $u$. Hence the dimension reduction theorem applies (see [Ch, Theorem 2.3]) and we conclude that the parabolic Hausdorff dimension of $\Sigma$ is at most $n$. Furthermore, it also implies that

$$
\operatorname{dim}_{\mathcal{H}}\{x \in \Omega:|\nabla u(x, t)|=0\} \leq n-2 .
$$

\section{Proof of Almgren's formula}

Here we present the proof of Almgren's monotonicity formula which works in our setting. Recall that $G_{t}=\Delta G$ and

$$
\nabla G=-\frac{x}{2\left(t+t_{0}\right)} G
$$


Compute

$$
\begin{aligned}
\frac{d}{d t} H(t) & =\int_{\mathbb{R}^{n}} \frac{d}{d t}\left[\sum_{k=1}^{m} u_{k}^{2}(x, t) G(x, t) d x\right]=\int_{\mathbb{R}^{n}}\left[2 u u_{t} G(x, t)+u^{2} G_{t}\right] d x \\
& =\int_{\mathbb{R}^{n}}\left[2 u u_{t} G(x, t)+u^{2} \Delta G\right] d x=\int_{\mathbb{R}^{n}}\left[2 u u_{t} G-2 u \nabla u \cdot \nabla G\right] d x \\
& =\int_{\mathbb{R}^{n}} 2 u\left[u_{t}+\frac{\nabla u}{2\left(t+t_{0}\right)}\right] G(x, t) d x .
\end{aligned}
$$

Next we transform $D(t)$,

$$
\begin{aligned}
\int_{\mathbb{R}^{n}}\left|\nabla u_{i}\right|^{2} G d x & =\int_{\mathbb{R}^{n}} \nabla u_{i} \nabla u_{i} G d x=-\int_{\mathbb{R}^{n}}\left[u_{i} \Delta u_{i} G+\nabla u_{i} \nabla G\right] d x \\
& =\int_{\mathbb{R}^{n}} u_{i}\left[u_{t}+\frac{x \cdot \nabla u_{i}}{2\left(t+t_{0}\right)}\right] G d x .
\end{aligned}
$$

Summing up with respect to all $i=1, \ldots, m$ we get

$$
D(t)=\int_{\mathbb{R}^{n}} u\left[u_{t}+\frac{\nabla u \cdot x}{2\left(t+t_{0}\right)}\right] G d x
$$

where

$$
u \nabla u \cdot x=\sum_{i, j} u_{i} D_{j} u_{i} x_{j} .
$$

Finally, to compute $\frac{d}{d t} D(t)$ we use the Rellich-Nečas identity

$$
\begin{aligned}
\operatorname{div}\left(\nabla G\left(\nabla u_{i}\right)^{2}\right)-2 \operatorname{div}( & \left.\left(\nabla u_{i} \cdot \nabla G\right) \cdot \nabla u_{i}\right) \\
= & \Delta G\left|\nabla u_{i}\right|^{2}-2\left(\nabla^{2} G \nabla u_{i}\right) \cdot \nabla u_{i}-2 \nabla u_{i} \cdot \nabla G \Delta u_{i} .
\end{aligned}
$$

Hence after integration

$$
\begin{aligned}
\int_{\mathbb{R}^{n}} \Delta G\left|\nabla u_{i}\right|^{2} & =2 \int_{\mathbb{R}^{n}}\left(\nabla^{2} G \nabla u_{i}\right) \nabla u_{i}+2 \int_{\mathbb{R}^{n}} \nabla u_{i} \nabla G \Delta u_{i} \\
= & 2 \int_{\mathbb{R}^{n}}\left(\left[-\frac{\mathrm{Id}}{2\left(t+t_{0}\right)}+\frac{x \otimes x}{4\left(t+t_{0}\right)^{2}}\right] \nabla u_{i}\right) \cdot \nabla u_{i} G+2 \int_{\mathbb{R}^{n}} \nabla u_{i} \nabla G \Delta u_{i} \\
= & 2 \int_{\mathbb{R}^{n}}\left(\frac{\nabla u_{i} \cdot x}{2\left(t+t_{0}\right)}\right)^{2} G-\frac{1}{t+t_{0}} \int_{\mathbb{R}^{n}}\left|\nabla u_{i}\right|^{2} \cdot G \\
& -2 \int_{\mathbb{R}^{n}} \frac{\nabla u_{i} \cdot x}{2\left(t+t_{0}\right)} \Delta u_{i} G d x, \quad i=1, \ldots, m .
\end{aligned}
$$

On the other hand,

$$
\begin{aligned}
\frac{d}{d t} D(t) & =\int_{\mathbb{R}^{n}} \frac{d}{d t}\left[\left(\sum D_{j} u_{i}\right)^{2} G\right] d x=\int_{\mathbb{R}^{n}} \frac{d}{d t}\left(\sum_{i=1}^{m}\left|\nabla u_{i}\right|^{2} G\right) d x \\
& =\sum_{i=1}^{m} \int_{\mathbb{R}^{n}} \frac{d}{d t}\left(\left|\nabla u_{i}\right|^{2} G\right) d x=\sum_{i=1}^{m} \int_{\mathbb{R}^{n}}\left[2 \nabla u_{i} \nabla\left(u_{i}\right)_{t} G+\left|\nabla u_{i}\right|^{2} G_{t}\right] d x
\end{aligned}
$$




$$
\begin{aligned}
= & \sum_{i=1}^{m} \int_{\mathbb{R}^{n}}\left\{2\left[-\left(u_{i}\right)_{t}\left(\Delta u_{i} \cdot G+\nabla u_{i} \nabla G\right)\right]+\left|\nabla u_{i}\right|^{2} G_{t}\right\} d x \\
= & \sum_{i=1}^{m} \int_{\mathbb{R}^{n}}\left(2 \sum\left[\left(u_{i}\right)_{t}^{2}+\left(u_{i}\right)_{t} \frac{\nabla u_{i} \cdot x}{2\left(t+t_{0}\right)}\right] G+\left|\nabla u_{i}\right|^{2} \Delta G\right) d x \\
= & \sum_{i=1}^{m}\left(\int_{\mathbb{R}^{n}} 2\left[\left(u_{i}\right)_{t}^{2}+\left(u_{i}\right)_{t} \frac{\nabla u_{i} \cdot x}{2\left(t+t_{0}\right)}\right] G\right. \\
& +2 \int_{\mathbb{R}^{n}}\left(\frac{\nabla u_{i} \cdot x}{2\left(t+t_{0}\right)}\right)^{2} G-\frac{1}{t+t_{0}} \int_{\mathbb{R}^{n}}\left|\nabla u_{i}\right|^{2} G \\
& \left.+2 \int_{\mathbb{R}^{n}} \frac{\nabla u_{i} \cdot x}{2\left(t+t_{0}\right)}\left(u_{i}\right)_{t} G d x\right) \\
= & 2 \sum_{i=1}^{m} \int_{\mathbb{R}^{n}}\left[\left(u_{i}\right)_{t}+\frac{\nabla u_{i} \cdot x}{2\left(t+t_{0}\right)}\right]^{2} G-\frac{1}{t+t_{0}} D(t) .
\end{aligned}
$$

Combining all these computations we have

$$
\begin{aligned}
\frac{d}{d t} N(t)=\frac{D(t)}{H(t)}+\frac{\left(t+t_{0}\right) \frac{d}{d t} D(t)}{H(t)}-\frac{\left(t+t_{0}\right) D(t) \frac{d}{d t} H(t)}{H^{2}(t)} \\
=\frac{t+t_{0}}{H^{2}(t)}\left[\frac{D(t) H(t)}{t+t_{0}}+H(t) \frac{d}{d t} D(t)-D(t) \frac{d}{d t} H(t)\right] \\
=\frac{t+t_{0}}{H^{2}(t)}\left[\left(2 \sum_{i=1}^{m} \int_{\mathbb{R}^{n}}\left[\left(u_{i}\right)_{t}+\frac{\nabla u_{i} \cdot x}{2\left(t+t_{0}\right)}\right]^{2}-\frac{d}{d t} D(t)\right) H(t)\right. \\
\left.\quad+H(t) \frac{d}{d t} D(t)-D(t) \cdot 2 \int_{\mathbb{R}^{n}} u\left[u_{t}+\frac{D u \cdot x}{2\left(t+t_{0}\right)}\right] G d x\right] \\
=2 \frac{t+t_{0}}{H^{2}(t)}\left[\int_{\mathbb{R}^{n}} u^{2} G \sum_{i=1}^{m} \int_{\mathbb{R}^{n}}\left[\left(u_{i}\right)_{t}+\frac{D u_{i} \cdot x}{2\left(t+t_{0}\right)}\right]^{2}-\left(\int_{\mathbb{R}^{n}} u\left[u_{t}+\frac{\nabla u \cdot x}{2\left(t+t_{0}\right)}\right] G\right)^{2}\right]
\end{aligned}
$$

where the last line follows from a simple observation that

$$
D(t)=\sum_{i=1}^{m} \int_{\mathbb{R}^{n}}\left|\nabla u_{i}\right|^{2} G=\int_{\mathbb{R}^{n}} u \cdot\left[u_{t}+\frac{\nabla u \cdot x}{2\left(t+t_{0}\right)}\right] G d x .
$$

Then from the Cauchy-Schwarz inequality we have $N^{\prime}(t) \geq 0$.

Remark. It is important to point out that if $N(t)=$ const then $u_{t}+\frac{\nabla u \cdot x}{2\left(t+t_{0}\right)}=c(t) u$. As we showed earlier, $c(t)$ is, in fact, the degree of homogeneity.

Theorem 32. Assume that $u=\left(u_{1}, \ldots, u_{m}\right)$ is the solution to our free boundary problem. Then $N(t)$ is nondecreasing.

Proof. Let us look back at those parts of the previous computations which contain integration by parts. Let $S$ be the zero set of $\nabla u$, and $S_{\varepsilon}$ its $\varepsilon$-neighborhood. 
Furthermore, let $\delta>0$ and

$$
u_{i}^{\delta}=\left(u_{i}-\delta\right)^{+} .
$$

In equations (11.1) and (11.2) after integration by parts we have to deal with the term

$$
\int_{\mathbb{R}^{n}} u_{i}^{\delta}\left(\Delta u_{i}^{\delta}+\left(u_{i}^{\delta}\right)_{t}\right) G d x+\underbrace{\int u_{i}^{\delta} \nabla u_{i}^{\delta} \cdot G d A_{i, \delta}}_{\text {boundary term }}
$$

where $d A_{i, \delta}$ is the area measure on the $\delta$-level surface of $u_{i}$. Both terms are welldefined and go to zero as $\delta \rightarrow 0$.

The next term that we have to deal with comes from the Rellich-Nečas identity. More precisely, it consists of two parts:

$$
\begin{aligned}
& I_{1}=\int_{\mathbb{R}^{n}} \nabla u_{i}^{\delta} \cdot x\left[\Delta u_{i}^{\delta}+\left(u_{i}^{\delta}\right)_{t}\right] G \\
& I_{2}=\int \nabla G \cdot\left|\nabla u_{i}^{\delta}\right|^{2} \cdot \vec{n} d A_{i, \delta}-2 \int\left(\nabla u_{i} \cdot \nabla G\right) \cdot \nabla u_{i} \cdot \vec{n} d A_{i, \delta},
\end{aligned}
$$

where $\vec{n}$ is the unit exterior normal to the $\delta$-level surface of $u_{i}$. Finally, we need to deal with the following term:

$$
\int_{\mathbb{R}^{n}} \nabla u_{i}^{\delta} \nabla\left(u_{i}^{\delta}\right)_{t} G=\int\left(u_{i}^{\delta}\right)_{t} \nabla u_{i}^{\delta} G d A_{i, \delta}-\int_{\mathbb{R}^{n}}\left(u_{i}^{\delta}\right)_{t}\left(\Delta u_{i}^{\delta} G+\nabla u_{i}^{\delta} \nabla G\right) .
$$

We thus need to estimate

$$
I_{3}=\int\left(u_{i}^{\delta}\right)_{t} \nabla u_{i}^{\delta} G \vec{n} d A_{i, \delta}-\int_{\mathbb{R}^{n}}\left(u_{i}^{\delta}\right)_{t}\left(\Delta u_{i}^{\delta}+\left(u_{i}^{\delta}\right)_{t}\right) \cdot G .
$$

These are all the "bad" terms that we are left with. First let us observe that on the boundary of $\Omega_{t}^{\delta}=\left\{u_{i}(x, t)>\delta\right\}, \nabla u_{i}^{\delta}=\left|\nabla u_{i}^{\delta}\right| \cdot \vec{n}$. Here we have

$$
\begin{aligned}
I_{1} & =\int_{\mathbb{R}^{n}} \nabla u_{i}^{\delta} \cdot x\left[\Delta u_{i}^{\delta}+\left(u_{i}^{\delta}\right)_{t}\right] G d x, \\
I_{2} & =\int \nabla u_{i}^{\delta} \cdot \nabla G\left|\nabla u_{i}^{\delta}\right| d A_{i, \delta}-2 \int \nabla u_{i}^{\delta} \cdot \nabla G\left|\nabla u_{i}^{\delta}\right| d A_{i, \delta} \\
& =-\int \nabla u_{i}^{\delta} \cdot \nabla G\left|\nabla u_{i}^{\delta}\right| d A_{i, \delta}=\int \frac{\nabla u_{i}^{\delta} \cdot x}{2\left(t+t_{0}\right)}\left|\nabla u_{i}^{\delta}\right| G d A_{i, \delta}, \\
I_{3} & =\int\left(u_{i}^{\delta}\right)_{t} \nabla u_{i}^{\delta} G d A_{i, \delta}-\int_{\mathbb{R}^{n}}\left(u_{i}^{\delta}\right)_{t}\left(\Delta u_{i}^{\delta}+\left(u_{i}^{\delta}\right)_{t}\right) G d x .
\end{aligned}
$$

Fix $\varepsilon$ and let $\delta \rightarrow 0$. Then the terms with $d A_{i, \delta}$ go to 0 (outside of $S_{\varepsilon}$ ). Since for $x$ near $S_{\varepsilon},|\nabla u|=o(1)$, we thus obtain

$$
I_{1}=o(1) \int_{\mathbb{R}^{n}}\left(\Delta u_{i}+\left(u_{i}\right)_{t}\right) G d x \underset{\varepsilon \rightarrow 0}{\longrightarrow} 0
$$

and similarly $I_{3} \underset{\varepsilon \rightarrow 0}{\longrightarrow} 0$. 


\section{References}

[A] F. J. Almgren, Jr. $Q$ valued functions minimizing Dirichlet's integral and the regularity of area minimizing rectifiable currents up to codimension two. Bull. Amer. Math. Soc. (N.S.) 8 (1983), 327-328.

[ACF $]$ H. W. Alt, L. A. Caffarelli, and A. Friedman, Variational problems with two phases and their free boundaries. Trans. Amer. Math. Soc. 282, (1984), 431-461.

[BS] S. P. Burke and T. E. W. Schuman, Diffusion flames. Industr. Engrg. Chem. 20 (1928), 998-1004.

[CL1] L. A. Caffarelli and F.-H. Lin, Singularly perturbed elliptic systems and multivalued harmonic functions with free boundaries. Preprint.

[CL2] L. A. Caffarelli and F.-H. Lin, An optimal partition problem for eigenvalues. J. Sci. Comput. 31 (2007), 5-18.

[CL3] L. A. Caffarelli and F.-H. Lin, in preparation.

[CP] L. A. Caffarelli and I. Peral, On $W^{1, p}$ estimates for elliptic equations in divergence form. Comm. Pure Appl. Math. 51 (1998), 1-21.

[CR] L. A. Caffarelli and J.-M. Roquejoffre, Uniform Hölder estimates in a class of elliptic systems and applications to singular limits in models for diffusion flames. Arch. Ration. Mech. Anal. 183 (2007), 457-487.

[CSa] L. A. Caffarelli and S. Salsa, A Geometric Approach to the Free Boundary Problems. Grad. Stud. Math. 68, Amer. Math. Soc., Providence, RI, 2005.

[CLLL] S.-M. Chang, C.-S. Lin, T.-C. Lin, and W.-W. Lin, Segregated nodal domains of two-dimensional multispecies Bose-Einstein condensates. Phys. D 196 (2004), 341-361.

[Ch] X.-Y. Chen, A strong unique continuation theorem for parabolic equations. Math. Ann. 311 (1998), 603-630.

[CTV1] M. Conti, S. Terracini, and G. Verzini, Nehari's problem and competing species systems. Ann. Inst. H. Poincaré Anal. Non Linéaire 19 (2002), 871-888.

[CTV2] M. Conti, S. Terracini, and G. Verzini, An optimal partition problem related to nonlinear eigenvalues. J. Funct. Anal. 198 (2003), 160-196.

[CTV3] M. Conti, S. Terracini, and G. Verzini, Asymptotic estimates for the spatial segregation of competitive systems. Adv. Math. 195 (2005), 524-560.

[CTV4] M. Conti, S. Terracini, and G. Verzini, A variational problem for the spatial segregation of reaction-diffusion systems. Indiana Univ. Math. J. 54 (2005), 779 815 .

[EFV] L. Escauriaza, F. J. Fernández, and S. Vessella, Doubling properties of caloric functions. Appl. Anal. 85 (2006), 205-223.

[GL] N. Garofalo and F.-H. Lin, Monotonicity properties of variational integrals, $A_{p}$ weights and unique continuation. Indiana Univ. Math. J. 35 (1986), 245-268.

[La] E. Landis, Second order equations of elliptic and parabolic type. Translations of Mathematical Monographs, 171. American Mathematical Society, Providence, RI, 1998.

[L1] F.-H. Lin, Static and moving vortices in Ginzburg-Landau theories. In: Nonlinear Partial Differential Equations in Geometry and Physics (Knoxville, TN, 
1995), Progr. Nonlinear Differential Equations Appl. 29, Birkhäuser, Basel, 1997, $71-111$.

[L2] F.-H. Lin, Nodal sets of solutions of elliptic and parabolic equations. Comm. Pure Appl. Math. 44 (1991), 287-308.

[LY] F.-H. Lin and X. Yang, Geometric Measure Theory. An Introduction. International Press, Boston, 2002.

[LSW] W. Littman, G. Stampacchia, and H. F. Weinberger, Regular points for elliptic equations with discontinuous coefficients. Ann. Scuola Norm. Sup. Pisa (3) 17 (1963), 43-77.

\title{
L. A. Caffarelli
}

Department of Mathematics

University of Texas at Austin

1 University Station - C1200

Austin, TX 78712-0257, USA

e-mail: caffarel@math.utexas .edu

\begin{abstract}
A. L. Karakhanyan
Department of Mathematics

University of Texas at Austin

1 University Station - C1200

Austin, TX 78712-0257, USA

e-mail: aram@math.utexas.edu
\end{abstract}

\section{Fang-Hua Lin}

Department of Mathematics

Courant Institute of Mathematical Sciences

New York University

251 Mercer Street

New York, NY 10012, USA

e-mail: linf@cims.nyu.edu

To access this journal online: www.birkhauser.ch/jfpta 